\title{
Artificial out-of-plane Ising antiferromagnet on the kagome lattice with very small farther-neighbor couplings
}

\author{
Jeanne Colbois $\odot,{ }^{1, *}$ Kevin Hofhuis $\odot,{ }^{2,3, \dagger}$ Zhaochu Luo $\odot,{ }^{2,3}$ Xueqiao Wang $\odot,{ }^{2,3}$ Aleš Hrabec $\odot,{ }^{2,3,4}$ \\ Laura J. Heyderman ${ }^{\circ},{ }^{2,3}$ and Frédéric Mila ${ }^{1}{ }^{1}$ \\ ${ }^{1}$ Institute of Physics, Ecole Polytechnique Fédérale de Lausanne (EPFL), CH-1015 Lausanne, Switzerland \\ ${ }^{2}$ Laboratory for Mesoscopic Systems, Department of Materials, ETH Zurich, 8093 Zurich, Switzerland \\ ${ }^{3}$ Laboratory for Multiscale Materials Experiments, Paul Scherrer Institute, 5232 Villigen PSI, Switzerland \\ ${ }^{4}$ Laboratory for Magnetism and Interface Physics, Department of Materials, ETH Zurich, 8093 Zurich, Switzerland
}

(Received 22 April 2021; revised 27 June 2021; accepted 28 June 2021; published 13 July 2021)

\begin{abstract}
Despite their simple formulation, short-range classical antiferromagnetic Ising models on frustrated lattices give rise to exotic phases of matter, in particular, due to their macroscopic ground-state degeneracy. Recent experiments on artificial spin systems comprising arrays of chirally coupled nanomagnets provide a significant strengthening of the nearest-neighbor couplings compared to systems with dipolar-coupled nanomagnets. This opens the way to design artificial spin systems emulating Ising models with nearest-neighbor couplings. In this paper, we compare the results of an extensive investigation with tensor network and Monte Carlo simulations of the nearest- and farther-neighbor $\left(J_{1}-J_{2}-J_{3 \|}\right)$ kagome Ising antiferromagnet with the experimental spinspin correlations of a kagome lattice of chirally coupled nanomagnets. Even though the ratios between the farther-neighbor couplings and the nearest-neighbor coupling estimated from micromagnetic simulations are much smaller than for dipolar-coupled nanomagnets, we show that they still play an essential role in the selection of the correlations.
\end{abstract}

DOI: 10.1103/PhysRevB.104.024418

\section{INTRODUCTION}

Artificial spin systems, or artificial spin ices, are arrays of mesoscopic single-domain nanomagnets whose moments can be probed individually using magnetic microscopy techniques. The moments interact either via their magnetostatic fields or, in the case of connected nanomagnets, via exchange interactions. The flexibility in the design of these arrays makes them extremely useful for the study of frustration and emergent Coulomb phases in two-dimensional systems [1-3]. The field has been continuously growing, showing great promise for the theory of frustrated systems, for experimental work on reprogrammable magnonic crystals [4,5], and for applications involving computation to implement computational logic [6-8], as a natural support for artificial neural networks $[9,10]$, or for reprogrammable magnetic robots [11].

When viewed as toy models for frustrated spin systems, the artificial spin systems face the intrinsic difficulty [4] that the physics that one would like to study-collective phenomena and exotic emergent models-is exactly that which is hard to explore via the single spin-flip dynamics available either through demagnetization protocols $[2,12,13]$ or thermal activity [14-19]. Additionally, the disorder of the arrays and the large size of the nanomagnets compared to the lattice spacing could, in principle, affect the simplicity of the modeling with Ising Hamiltonians. Despite these difficulties, great success has been achieved by describing artificial spin systems based

\footnotetext{
*jeanne.colbois@epfl.ch

${ }^{\dagger}$ kevin.hofhuis@psi.ch
}

on arrays of nanomagnets using Ising Hamiltonians with longrange, dipolar couplings. Two prominent examples are the artificial dipolar kagome spin ice [15], for which Monte Carlo simulations predict a series of magnetic phase transitions $[20,21]$, and the artificial dipolar kagome Ising antiferromagnet $[22,23]$, where a weakly first-order phase transition to a long-range ordered ground state has been established [23-25]. The quest for a true ground-state degeneracy achieved in artificial spin ices reached an important step when a proposal from Möller and Moessner to recover the macroscopic ground-state degeneracy of spin ice on the square lattice [26] was successfully realized $[4,27,28]$. On the kagome lattice, however, the quest is still open. To control the couplings between the nanomagnets, new possibilities are being explored, such as the introduction of mixed anisotropy [29] or of a soft ferromagnetic underlayer below the moments [30]. In the present paper, motivated by the design of arrays of chirally coupled nanomagnets [29] promising the implementation of strong nearest-neighbor couplings, we numerically explore nearest- and farther- neighbor Ising antiferromagnets on the kagome lattice and compare our theoretical results to those of an experiment on an artificial array of chirally coupled nanomagnets.

The difficulties with farther-neighbor frustrated spin systems are not restricted to experiments; it is also challenging to study them numerically using Monte Carlo simulations (see, for instance, the dipolar kagome Ising model $[23,24])$, since ad hoc updates corresponding to the collective dynamics of the specific model are often needed to combat frustration and critical slowing down (see, for instance, Ref. [31]). Another technique has shown promising results in the study of classical 
statistical mechanics: since the pioneering work of Nishino and Okunishi [32], the transfer matrix techniques applied successfully to classical spin systems in the 1950s and 1980s have found a formulation in the modern language of tensor networks [33,34]. Partition functions, expressed as the trace of a transfer matrix, can be seen as the contraction of a 2D tensor network, which can be computed approximately using techniques initially developed for the study of 1D quantum systems $[35,36]$ or techniques designed specifically for $2 \mathrm{D}$ classical problems [32,37-39]. Of particular interest to us, tensor networks have been used on the anisotropic kagome Ising antiferromagnet in a field [40], on 2D and 3D frustrated models to accurately determine their residual entropies [41], and more recently on a farther-neighbor frustrated model on the kagome lattice [42]. In this paper, we apply tensor networks as a useful complement to Monte Carlo simulations for the study of the relevant farther-neighbor Ising models.

Continuing on the work of Ref. [29], we consider a kagome lattice of chirally coupled nanomagnets. The main idea is to rely on interfacial Dzyaloshinskii-Moriya interactions (DMI) to create regions with out-of-plane anisotropy (OOP regions) connected by chiral domain walls to regions with in-plane anisotropy (IP regions). Arranging the OOP regions on a kagome lattice and separating them with IP regions, one can then use the IP regions to create a very strong nearest-neighbor antiferromagnetic coupling between OOP regions. At first sight, one could expect the system to behave simply as a nearest-neighbor Ising antiferromagnet on the kagome lattice. In Sec. II, we describe this experiment in detail and perform micromagnetic simulations. The effective antiferromagnetic Ising model for the OOP regions that we obtain has very strong nearest-neighbor couplings and relatively weak nextand next-next-nearest-neighbor couplings-much smaller than in the dipolar case. In the remainder of the paper, we progressively build toward this farther-neighbor model, providing tensor network and Monte Carlo data for the spin-spin correlations of a series of short-range Ising models on the kagome lattice, and comparing these correlations to the experimental results. We start in Sec. III with the nearest-neighbor Ising antiferromagnet on the kagome lattice, which was extensively studied before the present paper [22,23,40,43-48]. We discuss its short-range spin-spin correlations and we compute its correlation length using tensor networks. We find that even when considering a magnetic field, and despite the strong nearest-neighbor couplings in the experiment, this model does not qualitatively explain the experimental observations. In Sec. IV, we show that even weak second-nearest-neighbor couplings can partially solve the issue by changing the order of magnitude of the next-nearest-neighbor correlations as compared to the next-next-nearest-neighbor correlations. We consider the effective farther-neighbor model in a field in Sec. V, and finally give an overall discussion of the results in Sec. VI.

\section{EXPERIMENT: A KAGOME LATTICE OF CHIRALLY COUPLED NANOMAGNETS}

We begin with a discussion of the experiments. They rely on the interfacial DMI [49-52],

$$
H_{\mathrm{DMI}}=\mathbf{D}_{i, j} \cdot\left(\mathbf{S}_{i} \times \mathbf{S}_{j}\right),
$$

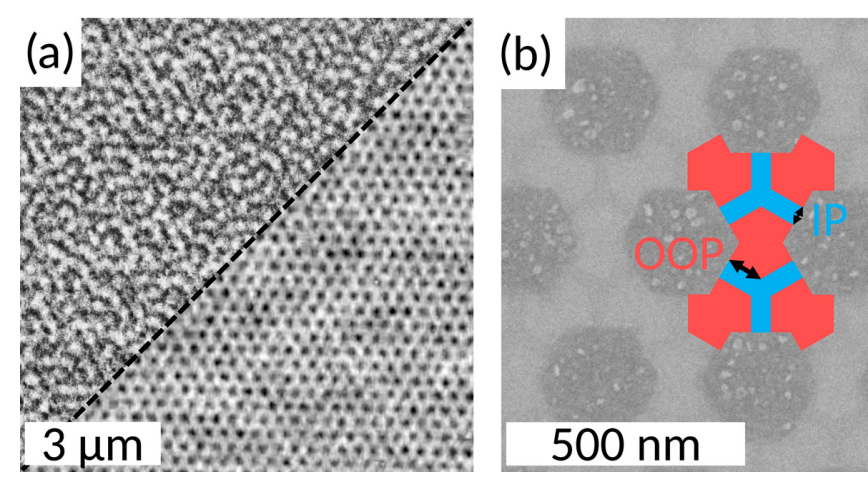

FIG. 1. The samples are extended kagome lattices etched from $\operatorname{Pt}(6 \mathrm{~nm}) / \mathrm{Co}(1.6 \mathrm{~nm}) / \operatorname{Al}(2 \mathrm{~nm})$ films. (a) Top left: Magnetic force microscopy (MFM) image of a sample. The black/white contrast shows the up/down magnetization of the regions with OOP anisotropy. Bottom right: AFM image of the sample structure. (b) Scanning electron microscopy image. Local Al oxidation can be seen as a grey contrast difference. A schematic of the out-of-plane (OOP) and in-plane (IP) regions is shown. The OOP (red) and IP (blue) anisotropy regions are defined by an OOP edge length of $100 \mathrm{~nm}$ and IP region width of $50 \mathrm{~nm}$. The OOP center-to-center distance is then geometrically set and is $\sqrt{3}$ OOP edge length + IP width.

which is allowed in environments with a lack of inversion symmetry and is induced by spin-orbit coupling. As discussed in Ref. [29], this interaction can be leveraged to induce an effective antiferromagnetic coupling between two OOP regions with well-defined magnetic anisotropy, which take on the role of Ising spins.

\section{A. Experimental setup \\ 1. Concept}

To create these effective antiferromagnetic couplings, we deposit a magnetic trilayer of $\mathrm{Pt}, \mathrm{Co}$ and $\mathrm{Al}$. These trilayers have a large DMI at the Pt/Co interface and their magnetic anisotropy can be tuned by oxidizing the AlOx. Through this oxidization, one can thus create regions of IP anisotropy and regions of OOP anisotropy and control the location of the domain walls between them with left-handed chirality.

Consider a simple system where two regions with OOP anisotropy, which we model as Ising spins, are connected by a region with IP anisotropy. Because of the DMI, it is energetically favorable to have the Ising spins in an antiferromagnetic arrangement. In Ref. [29], it was shown that this antiferromagnetic alignment can be realized in Ising chains and on the square lattice after applying an external magnetic field and provided that the IP regions are small enough. A small kagome sample was also brought to a ground state of the antiferromagnetic nearest-neighbor Ising model, namely, an ice-rule obeying state. Here, we apply this procedure to larger kagome systems (Fig. 1).

\section{Details of the sample fabrication and demagnetization protocol}

The fabrication of the samples is performed using electron beam lithography. First, $\mathrm{Pt}(6 \mathrm{~nm}) / \mathrm{Co}(1.6 \mathrm{~nm}) / \mathrm{Al}(2 \mathrm{~nm})$ films are deposited on a 200-nm-thick SiNx layer on a silicon 
substrate by DC magnetron sputtering at a base pressure of $2 \times 10^{-8} \mathrm{mbar}$ and at a deposition pressure of $3 \times 10^{-3} \mathrm{mbar}$. After patterning a polymethyl methacrylate (PMMA) mask with an electron beam writer, the trilayers are milled with Ar ions. The anisotropy of the various regions is determined by whether the Al layer is covered by the mask: the future IP regions are protected by $2 \mathrm{~nm} \mathrm{Ta}$, while the future OOP regions are exposed to oxygen plasma at a power of $30 \mathrm{~W}$ and an oxygen pressure of 0.013 mbar. For statistics, 20 samples of size $20 \times 20 \mu \mathrm{m}^{2}$ are patterned on the same substrate. With an IP length of $50 \mathrm{~nm}$ and an OOP edge length of $100 \mathrm{~nm}$, this amounts to approximately 2300 sites per sample.

All 20 samples have been AC demagnetized by a fieldbased protocol of sinusoidal OOP fields with decreasing amplitudes. The sinusoid amplitude decreased from 900 Oe to 30 Oe linearly in 4000 steps over a period of two hours. At each step, a single sinusoidal period is applied with a frequency of $2 \mathrm{~Hz}$. Several demagnetization protocols were tested, as discussed in Appendix A.

\section{Magnetic force microscopy}

Magnetic force microscopy (MFM) measurements are performed after the demagnetization protocol to extract the OOP spin configurations. A protective layer of PMMA is spin coated on each sample for the MFM measurements. The MFM tips, coated by $\mathrm{CoCr}$, are sensitive to magnetic stray fields. The tip is scanned over the sample at a frequency of $1 \mathrm{~Hz}$ and 512 pixels/line. An example of the resulting phase contrast is given in Fig. 1. The contrast gives information about the OOP magnetization, with white (black) for down (up) magnetized OOP regions. The low thickness of magnetic material makes the highly sensitive MFM tip a good candidate for imaging the stray fields of the samples.

\section{B. Micromagnetic simulations and effective model}

As we will discuss in Secs. III and IV, the results of tensor networks and Monte Carlo simulations strongly suggest that farther-neighbor couplings are relevant in this experiment. We see two possible sources of such couplings: the simple dipolar interaction between the regions with OOP anisotropy, and a possible interaction (of dipolar nature or some more subtle origin) between the regions with IP anisotropy. Here, we determine the likelihood of these scenarios by performing micromagnetic simulations [53-57].

Our aim is to find an effective Hamiltonian for the OOP regions, which are modeled with Ising variables. Indeed, it is a priori unclear how to combine the IP region and the DMI interactions with the dipolar interactions to give an effective model for the Ising spins. We therefore perform micromagnetic simulations for islands (OOP and IP regions) with three and five sites to determine these effective interactions. For this, we consider the most generic spin model for five sites, including three-, four-, and five-site interactions, respecting the cluster symmetries. In the micromagnetic computations, we did not find any evidence suggesting $\mathbb{Z}_{2}$ symmetry breaking. The effective Hamiltonian thus reduces to the following expression, where this symmetry is explicitly imposed by

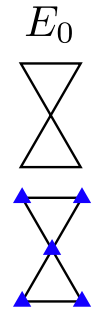

I (2)

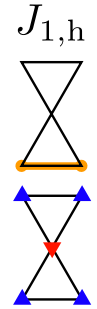

II (2)

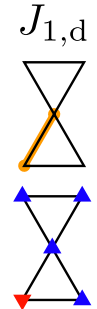

III (8)

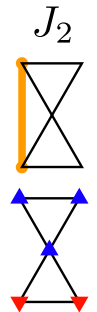

IV (4)
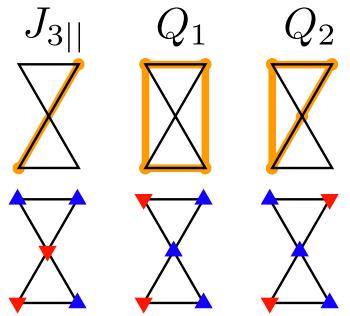

$\mathrm{V}(8)$

VI (4) VII (4)
FIG. 2. The first row indicates the couplings for the generic model with five sites of Eq. (2) (the symmetries of the system and the $\mathbb{Z}_{2}$ symmetry being imposed). The orange lines indicate the group of spins involved in the interaction corresponding to a given coupling. If the symmetries are imposed, some configurations will have the same energy; we can therefore group configurations by their energy. The second row shows one configuration (blue triangles for up spins and red triangles for down spins) for each of these seven different groups. Each group is indexed by a Roman number and the Arabic numbers indicate how many configurations are in each group.

removing terms with an odd number of spins:

$$
\begin{aligned}
H= & E_{0}+J_{1, \mathrm{~h}} \sum_{\langle i, j\rangle_{1, \mathrm{~h}}} \sigma_{i} \sigma_{j}+J_{1, \mathrm{~d}} \sum_{\langle i, j\rangle_{1 d}} \sigma_{i} \sigma_{j} \\
& +J_{2} \sum_{\langle i, j\rangle_{2}} \sigma_{i} \sigma_{j}+J_{3 \|} \sum_{\langle i, j\rangle_{3 \|}} \sigma_{i} \sigma_{j} \\
& +Q_{1} \sum_{\langle i, j, k, l\rangle_{1}} \sigma_{i} \sigma_{j} \sigma_{k} \sigma_{l}+Q_{2} \sum_{\langle i, j, k, l\rangle_{2}} \sigma_{i} \sigma_{j} \sigma_{k} \sigma_{l},
\end{aligned}
$$

and where the groups of spins to which the couplings apply are defined in Fig. 2. The $J$ couplings correspond to the usual pair interactions while the $Q$ couplings correspond to four-site interactions.

\section{Micromagnetic simulation parameters}

We performed micromagnetic simulations using $\mathrm{MuMax}^{3}$ (v3.10) $[56,57]$ to determine the (relative) coupling constants of the chirally coupled nanomagnet geometry. The simulations are carried out with a cell size of 0.866 by 0.866 by 1.6 $\mathrm{nm}^{3}$ (1.6 nm is shorter than the exchange length) and with 2048 by 2048 by 1 simulation world size. The cell sizes are chosen such that the angled edges are accurately simulated.

Typical material parameters for cobalt (Co) are used: a saturation magnetization $M_{\mathrm{s}}=600 \mathrm{kA} / \mathrm{m}$, an exchange stiffness $A=16 \times 10^{-12} \mathrm{~J} / \mathrm{m}$, and a magnetocrystalline anisotropy $K_{u}=570 \mathrm{~kJ} / \mathrm{m}^{3}$ in the OOP region and $K_{u}=0$ in the IP region. Interfacial DMI is introduced by setting $D_{\text {ind }}=0.9 \pm 0.1 \mathrm{~mJ} / \mathrm{m}^{2}$ [29]. The Gilbert damping $\alpha$ is set to 1.0 to allow the simulation to relax quickly to the state we are interested in, i.e., the state after energy minimization. The various coupling constants follow from simulating different $\mathrm{OOP}$ and IP configurations.

\section{Results of the micromagnetic simulations}

We first simulate the system in the absence of regions with IP anisotropy, where the interactions are of a dipolar nature, similar to Ref. [23]. The detail of the results for each 
configuration of Fig. 2 can be found in Appendix B. We find that

$$
\begin{aligned}
& J_{1}^{\mathrm{dip}}=(1.87 \pm 0.01) \times 10^{-20} \mathrm{~J}, \\
& \frac{J_{2}^{\mathrm{dip}}}{J_{1}^{\mathrm{dip}}}=0.1188 \pm 0.0006, \\
& \frac{J_{3 \|}^{\text {dip }}}{J_{1}^{\text {dip }}}=0.0769 \pm 0.0003,
\end{aligned}
$$

where the errors are dominated by the error on $J_{1}$-we find two slightly different values for $J_{1, \mathrm{~d}}$ and $J_{1, \mathrm{~h}}$ [58]. $Q_{1}$ and $Q_{2}$ are zero within the error bars. In this case, we indeed recover the dipolar couplings, with a factor of 1.6 correction to the nearest-neighbor coupling as compared to the point-dipole approximation, coming from the finite size of the elements and their proximity $[22,23])$.

We then consider the effect of the IP regions. A small subtlety has to be addressed in this case: a priori, for a given OOP configuration (labeled by roman numbers in Fig. 2), there can be several IP configurations. We proceed in two steps:

(1) For each configuration of three Ising spins on a triangle, we find the IP configuration that minimizes the energy (see Fig. 3). From this, we can already extract the nearestneighbor coupling

$$
J_{1}=(1.63 \pm 0.01) \times 10^{-19} \mathrm{~J},
$$

(for an IP width of $50 \mathrm{~nm}$ ) that is, almost one order of magnitude larger than in Eq. (3). The main source of the error is that the micromagnetic results are not completely rotationinvariant, a difficulty probably related to the use of a square grid for discretization in $\mathrm{MuMax}^{3}$.

(2) For each configuration of five Ising spins on a pair of triangles, we look for the combined configuration of the two IP regions minimizing the energy. The optimal configurations and their respective energies can be found in Appendix B. The effective model for the OOP region (Ising spins) is based on these energies.

It is important to note that we assume here that the IP region will take the configuration that locally minimizes the energy. Because of the limited MFM resolution and the small width of the IP regions, we have not been able to determine from the experimental scans whether this is actually the case.

We give the results for various IP widths and for various values of the DMI in Fig. 4. In particular, for an IP width of $50 \mathrm{~nm}$ and $D=0.9 \mathrm{~mJ} / \mathrm{m}^{2}$, corresponding to our experiment, we find for the full $J_{1}$ coupling involving dipolar and IP-mediated interactions:

$$
J_{1}=(1.6 \pm 0.03) \times 10^{-19} \mathrm{~J} .
$$

This result, computed by minimizing the energy of pairs of triangles, is in agreement (within the error bars) with the result of the equality in Eq. (4) which was obtained by minimizing the energy of single triangles. For the farther-neighbor couplings, we find

$$
\frac{J_{2}}{J_{1}} \cong 0.0235 \pm 0.0004
$$

(a)

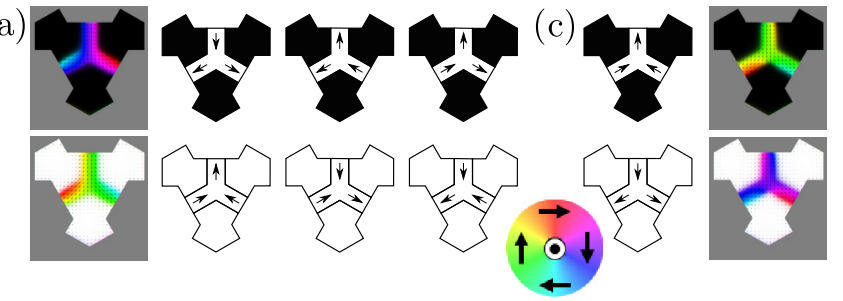

(b)

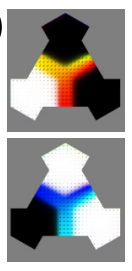

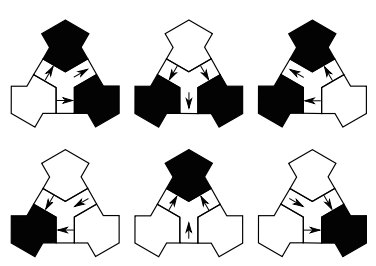

(d)

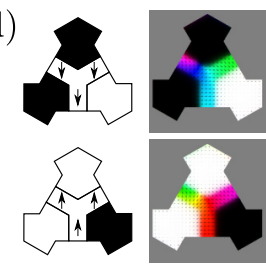

FIG. 3. Configurations of the regions with OOP anisotropy and IP anisotropy on a triangle. Black and white OOP regions correspond to up and down Ising spins, respectively. (a), (b) In the grey squares, the results of the micromagnetic simulations are given; these results are also schematically represented for readability. The colors in the IP region refer to the orientation of the local magnetization in the plane, as labeled by the colored disk. (a) Optimal configuration of the IP regions when all the Ising spins are aligned: one-in two-out for all up spins, two-in one-out for all down spins. These configurations have energy $E=-7.5588 \times 10^{-18} \pm 10^{-22} \mathrm{~J}$. (b) Optimal configurations of the IP regions when one or two spins are up: the IP region points toward the up spins. These configurations have energy $E=-8.209 \times 10^{-18} \pm 5 \times 10^{-21} \mathrm{~J}$. (c), (d) Examples of IP configurations which give a higher energy for the OOP configurations corresponding to (a) and (b). Here, the schematic representation shows how the IP region was initialized. (c) A two-in one-out configuration for all up spins, or a one-in two-out configuration for all down spins, is not energetically favorable. (d) If, initially, the IP magnetization does not point toward the up spin(s), the system tries to relax to the IP configurations shown in (b).

and

$$
\frac{J_{3 \|}}{J_{1}} \cong 0.0103 \pm 0.0001
$$

We see that, as compared to the pure OOP model, when the IP region of width $50 \mathrm{~nm}$ is taken into account, the nearestneighbor coupling is increased by almost a factor of 10 , but that the farther-neighbor couplings are affected as well by a factor of

$$
\frac{J_{2}}{J_{2}^{\mathrm{dip}}} \cong 1.67 \pm 0.01
$$

and

$$
\frac{J_{3 \|}}{J_{3 \|}^{\text {dip }}} \cong 1.12 \pm 0.01,
$$

respectively. If the IP region had only contributed to the nearest-neighbor coupling (and not to farther-neighbor couplings), the $J_{2}^{\text {dip }}$ and $J_{3 \|}^{\text {dip }}$ couplings would have had to be compared not to $J_{1}^{\text {dip }}$ but to the full $J_{1}$. In this case, instead of the couplings in Fig. 4, for an IP width of $50 \mathrm{~nm}$, we would 


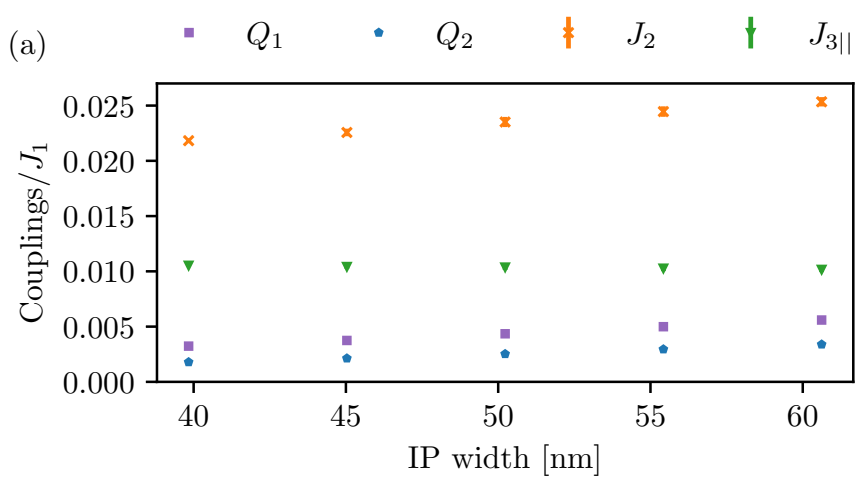

(b)

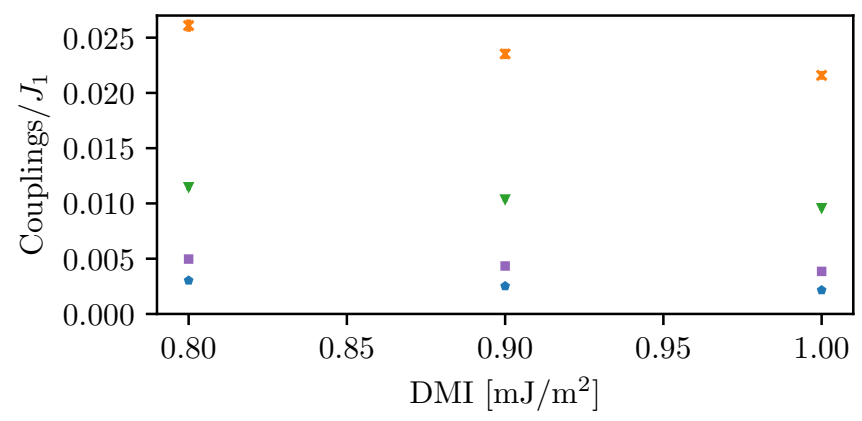

FIG. 4. The value of the couplings from Fig. 2, relative to $J_{1}$, as obtained from the micromagnetic configurations. Error bars for $J_{2} / J_{1}$ and $J_{3 \|} / J_{1}$ are computed from the error bars on $J_{1}$ and from the change in the value of $J_{2}$ and $J_{3 \|}$ when $Q_{1}$ and $Q_{2}$ are taken into account versus when they are not. (a) Variation of the couplings as a function of the width of the IP domain, with $D=0.9 \mathrm{~mJ} / \mathrm{m}^{2}$. (b) Variation of the couplings as a function of the DMI, with an IP width of $50 \mathrm{~nm}$.

have found that

$$
\begin{aligned}
& \frac{J_{2}^{\text {dip }}}{J_{1}}=0.0139 \pm 0.0002, \\
& \frac{J_{3 \|}^{\text {dip }}}{J_{1}}=0.0090 \pm 0.0001 .
\end{aligned}
$$

Thus, when the DMI and dipolar interactions are considered for a cluster of five sites, we find that the nearest-neighbor couplings are increased by a much larger factor than the second- and third-neighbor couplings, resulting in an effective model with very small farther-neighbor interactions as compared to the nearest-neighbor couplings. These results vary only slightly with the change in IP width or in DMI, as shown in Fig. 4.

\section{Sources of a magnetic field}

On top of the effective model discussed above, we have to consider the possible effect of a magnetic field. Indeed, there are two possible sources of a longitudinal field (i.e., a magnetic field parallel to the Ising spin axis) in the experiment: the offset of the demagnetization field and the stray field of the MFM measurement tip [59]. The saturation field of the samples (of the order of $4 J_{1}$ ) is estimated to be of the order of $2 \mathrm{kOe}$. The offset in the demagnetization field is of the order of 10 Oe and the stray fields from the MFM tip are of order

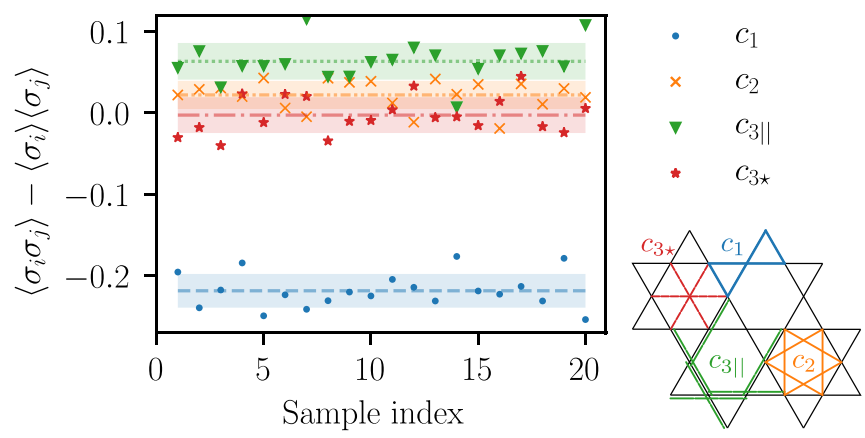

FIG. 5. Short-range spin-spin correlations of the 20 kagome samples. The considered spin pairs are defined in the inset. $c_{1}$ and $c_{2}$ correspond to nearest- and next-nearest-neighbor correlations, $c_{3 \|}$ and $c_{3 \star}$ to third neighbors. The dashed lines show the mean value and the highlighted areas correspond to one standard deviation around the average.

500 Oe. This means that if there is a field, we expect it to be of the order of $h \sim J_{2}$ to $h \sim J_{1}$.

\section{Experimental results}

To characterize the experimental results, we proceed as in Ref. [22] and we extract the experimental spin-spin correlations to compare them against the models under consideration. More precisely, for the four types of spin pairs illustrated in Fig. 5, we estimate the connected correlation functions

$$
\left\langle\sigma_{i} \sigma_{j}\right\rangle-\left\langle\sigma_{i}\right\rangle\left\langle\sigma_{j}\right\rangle
$$

using, for spin pairs of type $k$, the unbiased estimator

$$
c_{k}=\frac{M}{M-1}\left(\frac{1}{M} \sum_{(i, j)_{k}} \sigma_{i} \sigma_{j}-\frac{1}{M^{2}} \sum_{i:(i, j)_{k}} \sigma_{i} \sum_{j:(i, j)_{k}} \sigma_{j}\right),
$$

where $M$ is the number of spin pairs of type $k$ in the lattice and where $\sum_{(i, j)_{k}}$ denotes the sum over all spin pairs of type $k$. Note that we use the statistical field theory expressions: connected correlations means $\left\langle\sigma_{i} \sigma_{j}\right\rangle-\left\langle\sigma_{i}\right\rangle\left\langle\sigma_{j}\right\rangle$ and disconnected correlations means $\left\langle\sigma_{i} \sigma_{j}\right\rangle$. The results for each sample are shown in Fig. 5. In this figure, the shaded areas correspond to the values of the respective correlations that are within one standard deviation of the mean over the samples. These means are given in Table I. As shown in Fig. 5, the results vary significantly from one sample to the next. It is important to note that the descending order of the correlations is almost systematically $\left|c_{1}\right|>c_{3 \|}>c_{2}$, often with $c_{2} \gtrsim c_{3 \star}$. This qualitative result will drive our analysis.

Additionally, we consider two other observables which are characteristic of the experimental results. First, for the net magnetization of the samples, we get a result significantly different from zero: $m=0.19 \pm 0.05$; all the samples are

TABLE I. Experimental values for the first spin-spin correlations (see Fig. 5).

\begin{tabular}{lccc}
\hline \hline$c_{1}$ & $c_{2}$ & $c_{3 \|}$ & $c_{3 \star}$ \\
\hline$-0.218 \pm 0.02$ & $0.022 \pm 0.02$ & $0.063 \pm 0.02$ & $-0.003 \pm 0.02$ \\
\hline \hline
\end{tabular}


TABLE II. Experimental values for the proportion of triangles with a given net magnetization.

\begin{tabular}{lccc}
\hline \hline$r_{m_{\Delta}=+3}$ & $r_{m_{\Delta}=+1}$ & $r_{m_{\Delta}=-1}$ & $r_{m_{\Delta}=-3}$ \\
\hline $0.10 \pm 0.024$ & $0.60 \pm 0.03$ & $0.28 \pm 0.04$ & $0.015 \pm 0.008$ \\
\hline \hline
\end{tabular}

magnetized in the same direction. Second, in each sample there are $10 \%$ to $20 \%$ of ferromagnetic triangles (frustrated triangles, which do not respect the two-up one-down, twodown one-up ice rules); we therefore compute the proportion of triangles with a given magnetization (analogous to the often used charge definition but without introducing a sign). The results are given in Table II; overall, the proportion of ice-rulebreaking triangles is $r_{f r}=r_{m_{\Delta}=+3}+r_{m_{\Delta}=-3}=0.12 \pm 0.03$.

\section{NEAREST-NEIGHBOR MODEL}

Given the very strong nearest-neighbor couplings predicted by the micromagnetic simulations, we start by checking whether the experimental results from Sec. II can be understood using a purely nearest-neighbor model:

$$
H_{\mathrm{NN}}=J_{1} \sum_{\langle i, j\rangle} \sigma_{i} \sigma_{j}-h \sum_{i} \sigma_{i} .
$$

We begin by revisiting the well-known case in zero field using tensor networks. Because of the significant magnetization of the experimental results, we then consider how the picture is modified when the external magnetic field is turned on, a case which has been extensively studied [40,60-63] but for which we did not find data regarding the first spin-spin correlations as a function of temperature.

\section{A. Nearest-neighbor Ising antiferromagnet in zero field}

Introduced in Ref. [64], the kagome lattice is a natural playground for frustrated models. In particular, the nearestneighbor Ising antiferromagnet on this lattice is known to have no order at any temperature, exhibiting a macroscopic groundstate degeneracy with an extremely large residual entropy $S=$ $0.50183 \ldots$.. [43]. Later, it was shown by Sütö that the spin-spin correlations in the nearest-neighbor antiferromagnetic Ising model on the kagome lattice decay exponentially [44], and that

$$
\left|\left\langle S_{i} S_{j}\right\rangle\right| \leqslant 4 \times 0.74^{|i-j|}
$$

which implies an upper bound for the correlation length [65]:

$$
\xi \leqslant \xi_{\text {Sütö }}-1 / \ln 0.74=3.32109 .
$$

An exact result was obtained more recently for the correlation length between middle spins from transfer matrix computations using Toeplitz determinants [47]:

$$
\xi=-\frac{2}{\ln (10-\sqrt{96})} \cong 1.2506 \ldots
$$

It is well-known that the correlation length can be computed from the two leading eigenvalues of the transfer matrix
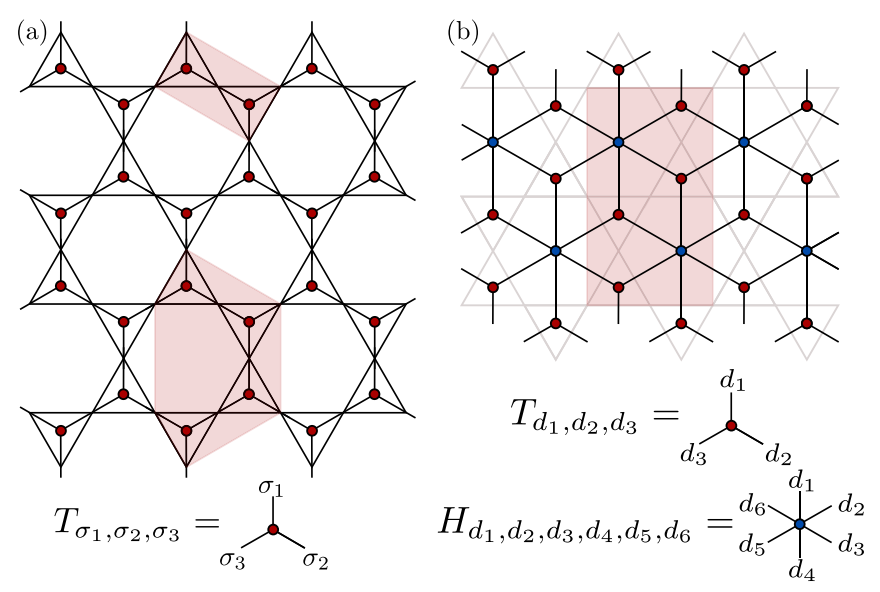

FIG. 6. Tensor network formulations for the partition function of the nearest-neighbor model on the kagome lattice. In highlight, we indicate the regions that can be grouped in a single tensor such that the tensor network on the honeycomb or dice lattice is reduced to a square lattice tensor network which can then be contracted using VUMPS. (a) Direct construction on the honeycomb lattice, with the tensor on the triangle given by Eq. (18), and which is easily extended to the nearest-neighbor model in a field. (b) Dual construction on the dice lattice, with the tensors given in Eqs. (20) and (21). This construction is easily extended to the next-nearest-neighbor model in zero field.

$\lambda_{1}, \lambda_{2}$ (see, for instance, Ref. [66]) as

$$
\frac{1}{\xi}=\ln \frac{\lambda_{2}}{\lambda_{1}}
$$

We write the partition function of the nearest-neighbor model on the kagome lattice as a tensor network as depicted in Fig. 6. We contract these networks using the variational uniform matrix product states (VUMPS) algorithm [36,67], which finds the leading eigenvector of the (infinite) 1D transfer matrix using as a variational ansatz a translationally invariant matrix product state (MPS).

In the direct construction, the tensor

$$
T_{\sigma_{i}, \sigma_{j}, \sigma_{k}}=e^{-\beta J_{1}\left(\sigma_{i} \sigma_{j}+\sigma_{j} \sigma_{k}+\sigma_{k} \sigma_{i}+1\right)}
$$

describes all the Boltzmann weights on a triangle, with a shift corresponding to the ground-state energy on a triangle. In the dual construction, we first define a classical dimer variable on each nearest-neighbor bond $\langle i, j\rangle$ :

$$
d_{i, j}:=\sigma_{i} \sigma_{j}
$$

When $d_{i, j}=1$, there is a dimer, and when $d_{i, j}=-1$, there is no dimer. The tensor on each triangle is thus

$$
T_{d_{i, j}, d_{j, k}, d_{k, i}}= \begin{cases}e^{-\beta J_{1}\left(d_{i, j}+d_{j, k}+d_{k, i}+1\right)} & d_{i, j} d_{j, k} d_{k, i}=1 \\ 0 & \text { otherwise. }\end{cases}
$$

The tensor on each hexagon $H_{d_{1}, d_{2}, d_{3}, d_{4}, d_{5}, d_{6}}$ only imposes that the number of dimers is even, such that the dimer configuration maps to a spin configuration:

$$
H_{d_{1}, d_{2}, d_{3}, d_{4}, d_{5}, d_{6}}= \begin{cases}1 & \text { if } d_{1} d_{2} d_{3} d_{4} d_{5} d_{6}=1 \\ 0 & \text { otherwise }\end{cases}
$$


TABLE III. First spin-spin correlations in the ground state of the nearest-neighbor model (see Fig. 7).

\begin{tabular}{lclll}
\hline \hline & $c_{1}$ & \multicolumn{1}{c}{$c_{2}$} & \multicolumn{1}{c}{$c_{3 \|}$} & \multicolumn{1}{c}{$c_{3 \star}$} \\
\hline Exact [45] & $-1 / 3$ & $0.1234 \ldots$ & $0.1014 \ldots$ & -0.0743 \\
Direct TN & $-1 / 3$ & 0.12343725 & 0.10144577 & -0.07480837 \\
Dual TN & $-1 / 3$ & 0.12343725 & 0.10144577 & -0.07480837 \\
\hline \hline
\end{tabular}

In both constructions, we recover the exact ground-state entropy of the kagome Ising antiferromagnet [43] to the 14th decimal place. For the first few correlations in the ground state, the two tensor constructions agree to the third decimal place with the exact results $[45,48]$ (Table III).

As a function of the temperature, the comparison between the tensor networks and the exact solution for the first few correlations is given in Fig. 7. We also use it as a benchmark for the Monte Carlo simulations, performed with a dual worm algorithm [68] (Appendix C). To extract the correlation length from the direct tensor network, we follow Ref. [69] and compute the first eigenvalues of the transfer matrix

$$
\lambda_{j}=e^{-\left(\epsilon_{j}+i \phi_{j}\right) P},
$$

with $\left|\lambda_{1}\right|>\left|\lambda_{2}\right|>\left|\lambda_{3}\right| \geqslant\left|\lambda_{4}\right| \geqslant \ldots$, where $\epsilon_{j}$ and $\phi_{j}$ correspond, respectively, to the log of the absolute value and to the phase, and where $P$ stands for the periodicity of the MPS in units of the number of lattice sites. The correlation length is thus given by

$$
\frac{1}{\xi}=\epsilon_{2}-\epsilon_{1}
$$

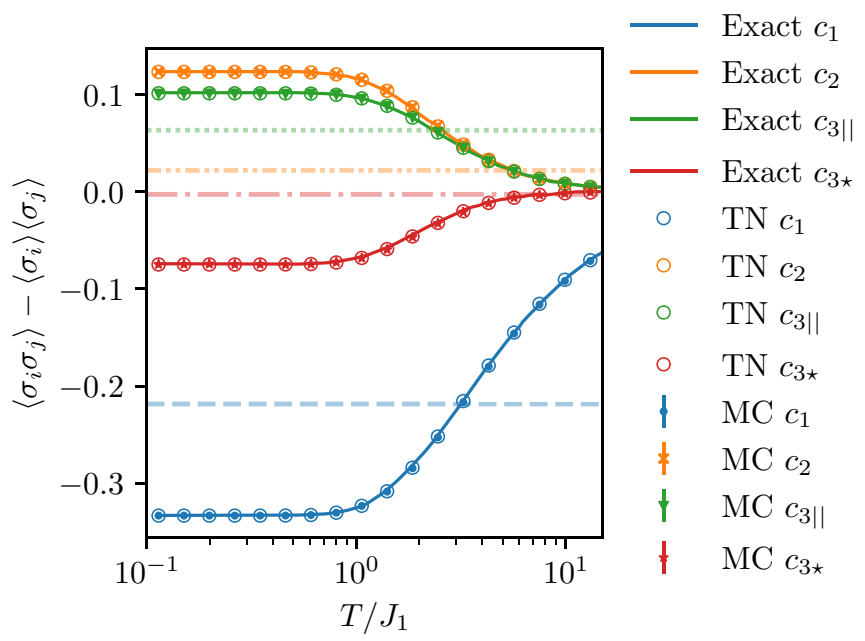

FIG. 7. First few correlations in the nearest-neighbor Ising antiferromagnet: comparison between the exact results (Ref. [45], extracted from Ref. [48]), and the experimental results. As a benchmark, we also show the results of the two numerical methods (TN: tensor networks, with (final) MPS bond dimension $\chi=13$, MC: Monte Carlo with number of sites $N=576$ ). The dashed lines correspond to the four experimental correlations results from Fig. 5. The results for the nearest-neighbor correlations are compatible with the experiment at relatively large effective temperatures $\left(T \sim 3 J_{1}\right)$ but the order of $c_{2}$ and $c_{3 \|}$ in the experiment is inverted as compared to the theoretical values in the nearest-neighbor model.

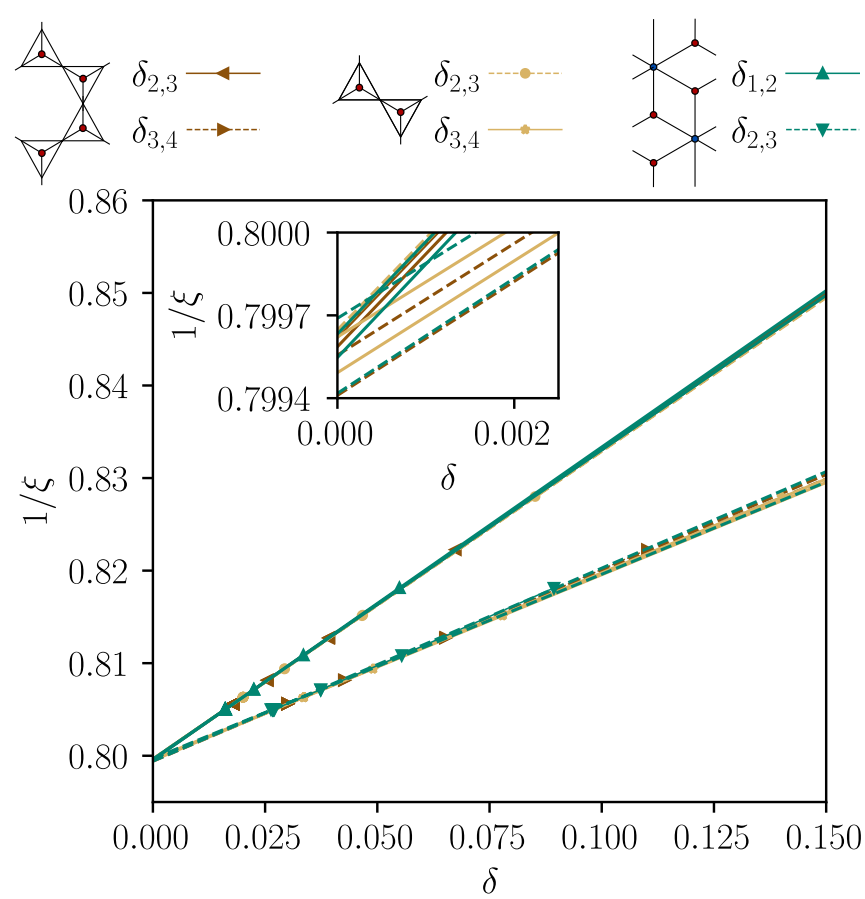

FIG. 8. For two slightly different tensor network constructions, we plot one over the correlation length [Eq. (23)] as a function of the logarithm of another gap in the transfer matrix spectrum [Eq. (27)]. The legend indicates, for each set of points, to which unit cell it is associated and which gap $\delta$ in the transfer matrix spectrum is selected. The correlation length corresponds to the correlations along a line of kagome lattice nearest neighbors. For each set of points, we do two fits, one with all the points and one without the largest $\delta$. The inset shows the crossing of each of these fit with the axis $\delta=0$, giving an estimate for the actual correlation length in the limit of an infinite bond dimension.

To extract the correlation length in the dual tensor network formulation, one can use the fact that the product of two spins is given by the product along a path of all the dimer variables separating them. We thus define a correlation tensor $C$ as the contraction along $d_{c}$ of the two tensors

$$
\begin{gathered}
T_{d_{\mathrm{top}, 2}, d_{r}, d_{c}}^{c}=d_{\mathrm{top}, 2} T_{d_{\mathrm{top}, 2}, d_{r}, d_{c}}, \\
H_{d_{\mathrm{top}, 1}, d_{c}, d_{3}, d_{4}, d_{5}, d_{6}}^{c}=d_{\mathrm{top}, 1} H_{d_{\mathrm{top}, 1}, d_{c}, d_{3}, d_{4}, d_{5}, d_{6}} .
\end{gathered}
$$

The correlation length is then given by

$$
\frac{1}{\xi}=\epsilon-\epsilon_{1},
$$

with $\lambda=e^{-(\epsilon+i \phi) P}$ corresponding to the leading eigenvalue of the transfer matrix based on $C$ (note that here, the periodicity of the MPS compared to the lattice is $P=2$ ). In both the direct and the dual cases, we define

$$
\delta_{i, j}=\epsilon_{j}-\epsilon_{i}
$$

the other gaps in the transfer matrix spectrum, which must go to zero in the limit of infinite bond dimensions to produce corrections to the form of the correlations [69]. In Fig. 8, the value of the correlation length as a function of such gaps 
for different bond dimensions is illustrated; in the direct construction, this is shown for two different orientations for the contractions displayed in Fig. 6. We are limited to a maximal bond dimension of $\chi=14$, after which the Schmidt values fall below numerical precision. From using the various constructions, selecting various gaps, and making the fits with all the points and all the points but one, we can finally extract the correlation length along the nearest-neighbor chains and the errors on its estimation as

$$
\xi=1.2507 \pm 0.0003
$$

in units of the lattice spacing. This is extremely short, consistent with the upper bond from Sütö's computation, and matches within the error bars the exact solution given in Eq. (16).

In Fig. 7, we compare the experimental results for the correlations to the nearest-neighbor Ising antiferromagnet at all temperatures. At this stage, we should recall that the samples are not at all expected to be thermally active. Here, the temperature is introduced as a Lagrange parameter for the energy in an attempt to account for the nonzero percentage of ice-rule-breaking triangles in a nonbiased way [70]. Accordingly, corresponding to the nonzero proportion of frustrated triangles, one can see that the experimental value for the nearest-neighbor correlations corresponds to a finite effective temperature.

The description with the nearest-neighbor model fails in two ways. First, because there is no $\mathbb{Z}_{2}$ symmetry breaking, the proportions of triangles of given magnetization (Table II) and the finite magnetization cannot be recovered. Second, the relative order of $c_{2}$ and $c_{3 \|}$ in the experiment is inverted as compared to the nearest-neighbor model: Despite the strong value of the nearest-neighbor couplings as compared to the farther-neighbor couplings from Fig. 4, considering only nearest-neighbor couplings does not allow for a valid qualitative description of the experiment from the point of view of the descending order of the spin-spin correlations.

\section{B. Nearest-neighbor Ising antiferromagnet in a field}

The experiments exhibit a finite magnetization and an imbalance of the number of triangles with a given magnetization. A simple way to account for this in the model is to introduce the corresponding Lagrange parameter, that is, the magnetic field. It is thus natural, as a next step, to test whether a longitudinal magnetic field lifting (partially) the groundstate degeneracy of the nearest-neighbor ground state could be enough to explain not only the magnetization and the proportion of triangles with a given magnetization but also the result that $c_{3 \|} \gtrsim 2 c_{2}$.

We use a tensor network contraction to compute the correlations systematically as a function of field and temperature. Before doing so, we take advantage of this construction to study the ground-state phase diagram of this kagome Ising antiferromagnet in a field. The ground state of the nearestneighbor model in a field [Eq. (13)] is known to exhibit a magnetization plateau $m=\frac{1}{3}$ for fields $0<\frac{h}{J_{1}}<4$, where each triangle bears two spins up and one spin down. The configurations in this plateau can be exactly mapped to a hardcore dimer model on the honeycomb lattice (placing a
TABLE IV. First spin-spin correlations in the critical ground state of the nearest-neighbor model in a field.

\begin{tabular}{lcccc}
\hline \hline & $c_{1}$ & $c_{2}$ & $c_{3 \|}$ & $c_{3 \star}$ \\
\hline Ref. [63] & $-4 / 9$ & 0.36756 & 0.07688 & -0.30396 \\
Direct TN $(\chi=80)$ & $-4 / 9$ & 0.36755 & 0.07689 & -0.30396 \\
\hline \hline
\end{tabular}

dimer on each down spin), leading to a macroscopic groundstate degeneracy with a residual entropy corresponding to one-third of the nearest-neighbor antiferromagnet on the triangular lattice [61-63,71-73]. The connected correlations are critical, decaying as $1 / r^{2}([63,73]$ and references therein). In the ground state, the correlations have been tabulated (Fig. 3 of Ref. [63]).

The model can be studied using the tensor network expression for the partition function from Fig. 6(a), with the slight modification that

$$
T_{\sigma_{1}, \sigma_{2}, \sigma_{3}}= \begin{cases}e^{-\beta J_{1}\left(\sum_{\langle i, j\rangle} \sigma_{i} \sigma_{j}+1\right)+\beta h\left(\sum_{i} \sigma_{i}-1\right) / 2} & h \leqslant 4 J_{1} \\ e^{-\beta J_{1}\left(\sum_{\langle i, j\rangle} \sigma_{i} \sigma_{j}-3\right)+\beta h\left(\sum_{i} \sigma_{i}-3\right) / 2} & h \geqslant 4 J_{1}\end{cases}
$$

(see as well Ref. [40] for a similar construction and a contraction with TRG, which was not applied to the case we present here). In the magnetization plateau, with bond dimension $\chi=80$, we find indeed

$$
S_{h<4 J_{1}}=0.1076886 \pm 10^{-7},
$$

which corresponds to one-third of the triangular Ising antiferromagnet entropy. We compare the correlations to the result of Ref. [63] in Table IV.

Similar to what happens on the square and triangular lattices [74,75], at the critical field $h / J_{1}=4$, a number of additional configurations contribute to the ground state. It has been noted before that at low temperature, this leads to a special value of the magnetization $m=3 / 5$ [76]. We find a slightly different value which is consistent between our tensor network computations in the ground state and our MC simulations at $T / J_{1}=7 \times 10^{-3}$ :

$$
\begin{gathered}
m_{h=4 J_{1}}^{\mathrm{TN}}=0.599660907836 \pm 10^{-12}, \\
m_{h=4 J_{1}}^{\mathrm{MC}}=0.59968 \pm 8 \times 10^{-5} .
\end{gathered}
$$

For the residual entropy, we find

$$
S_{h=4 J_{1}}=0.387800244253 \pm 10^{-12} \text {. }
$$

The value of the residual entropy is consistent with Ref. [76], although with a significant improvement in the precision, owing to the small bond dimension required to compute the entropy [77]. The values of the first few correlations at small but nonzero temperatures for $h / J_{1}=4$ are given in Table V, where it can be seen that the Monte Carlo and tensor network computations agree. A similar analysis as the one performed in zero field (Fig. 9) yields a finite correlation length

$$
\xi_{h=4 J_{1}}=0.8627 \pm 0.0001
$$

in units of the lattice spacing. This small correlation length is the reason why the tensor network results are obtained with such high precision even with extremely small bond dimensions (here, the largest bond dimension is $\chi=10$ as 
TABLE V. First spin-spin correlations in the ground state in the nearest-neighbor model in a longitudinal field $h=4 J_{1}$. For the tensor network, the Schmidt values decay extremely fast and fall below numerical precision for $\chi>10$. For the Monte Carlo, we show results with a number of sites $N=2304$, at temperature $T / J=7 \cdot 10^{-3}$.

\begin{tabular}{lcccl}
\hline \hline & $c_{1}$ & $c$ & $c_{2}$ & \multicolumn{1}{c}{$c_{3 \star}$} \\
\hline Direct TN & -0.1602714 & 0.04322263 & 0.0382691 & -0.019949696 \\
MC & $-0.16025(6)$ & $0.04322(8)$ & $0.03819(8)$ & $-0.0198(3)$ \\
\hline \hline
\end{tabular}

for larger bond dimensions, the Schmidt values decay below numerical precision). For $\frac{h}{J_{1}}>4$, the ground state is the fully ferromagnetic state.

We first check that by introducing a field, we can account for the number of triangles with a given magnetization. For this, we compute the proportions $r_{m_{\Delta}=+3}, r_{m_{\Delta}=+1}, r_{m_{\Delta}=-1}$, and $r_{m_{\Delta}=-3}$ for regularly spaced fields (every $0.2 J_{1}$ ). As shown in Fig. 10, we find that we obtain the best fit at $h=(1.6 \pm 0.2) J_{1}$ for a temperature $T=(2.8 \pm 0.2) J_{1}$. As a direct consequence, in this region, the magnetization and the nearest-neighbor correlations match the experimental results.

We compute the correlations as a function of field and temperature using the tensor network construction. For a few fields, we compute the spin-spin correlations with Monte Carlo simulations using replicas in field and temperature (similar to Refs. [76,79]) to confirm the tensor network results; this is plotted in Fig. 11 (a plot for the magnetization can be found in Appendix D). From the systematic computation of the spin-spin correlations, we find that at all fields the $c_{2}$ correlations remain larger or equal to the $c_{3||}$ correlations.

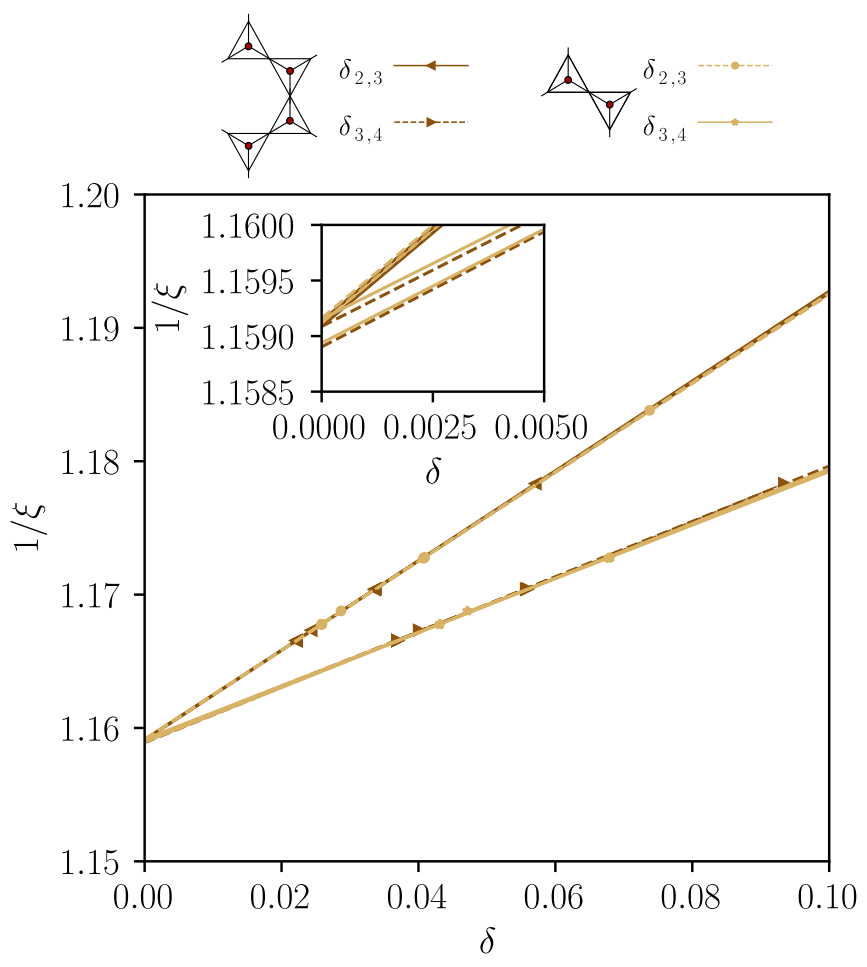

FIG. 9. Determining the correlation length Eq. (34) (for the connected correlations) at $h=4 J_{1}$. See the caption of Fig. 8 for details.

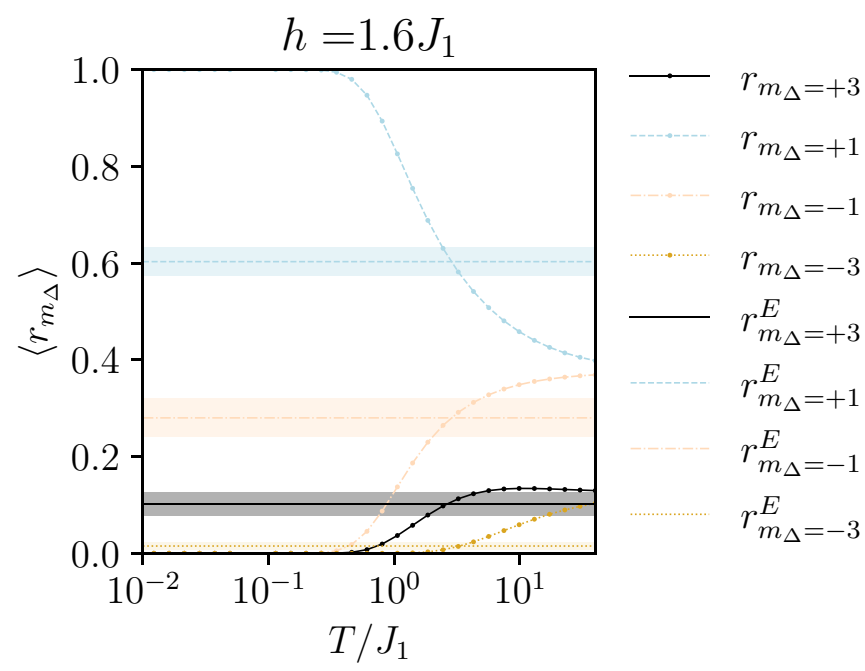

FIG. 10. Tensor network (maximal bond dimension $\chi=80$ ) results for the proportion of triangles of given magnetization at a field of $h=1.6 J_{1}$. For different fields spaced regularly every $0.2 J_{1}$, this is the one at which the prediction from the tensor network simulations is closest to the experimental results. The optimal temperature is $T=(2.8 \pm 0.2) J_{1}$. The data for the proportion of triangles of each type at regularly spaced fields can be found at Ref. [78].

In particular, at large temperatures, $c_{3 \|}$ is essentially equal to $c_{2}$. Thus, even though combining the magnetic field and the temperature allows us to account for the proportion of triangles of given magnetization, and therefore account for both the magnetization and the percentages of frustrated triangles, the nearest-neighbor model is not sufficient to fully account for the experimental results. This is possible because the proportion of each type of triangle is mostly related to the nearest-neighbor correlations as well as the magnetization, which are controlled, respectively, by $J_{1} / T$ and $h / T$, but the farther-neighbor spin-spin correlations are related to correlations between the triangles. This is somewhat surprising, since the next-nearest-neighbor coupling is only about $2 \%$ of $J_{1}$.

\section{NEXT-NEAREST-NEIGHBOR ISING ANTIFERROMAGNET}

Since the nearest-neighbor model does not fully describe the experimental correlations, and since the micromagnetic simulations predict a small but nonzero value for the nextnearest-neighbor couplings, we now consider whether such small couplings are sufficient to explain the inversion of the relative order of the $c_{2}$ and $c_{3||}$ correlations. Farther-neighbor couplings are known to lift the degeneracy either partially $[31,80,81]$ or completely $[80,82]$ (depending on whether they are ferro- or antiferromagnetic). For now, we ignore the problem of the magnetization and consider the Hamiltonian $[80,83]$

$$
H_{\mathrm{NNN}}=J_{1} \sum_{\langle i, j\rangle} \sigma_{i} \sigma_{j}+J_{2} \sum_{\langle i, j\rangle_{2}} \sigma_{i} \sigma_{j},
$$

where $\langle i, j\rangle_{2}$ stands for next-nearest-neighbor spin pairs as illustrated by $c_{2}$ in the inset of Fig. 5. The key question is then how large the next-nearest-neighbor coupling has to be to 


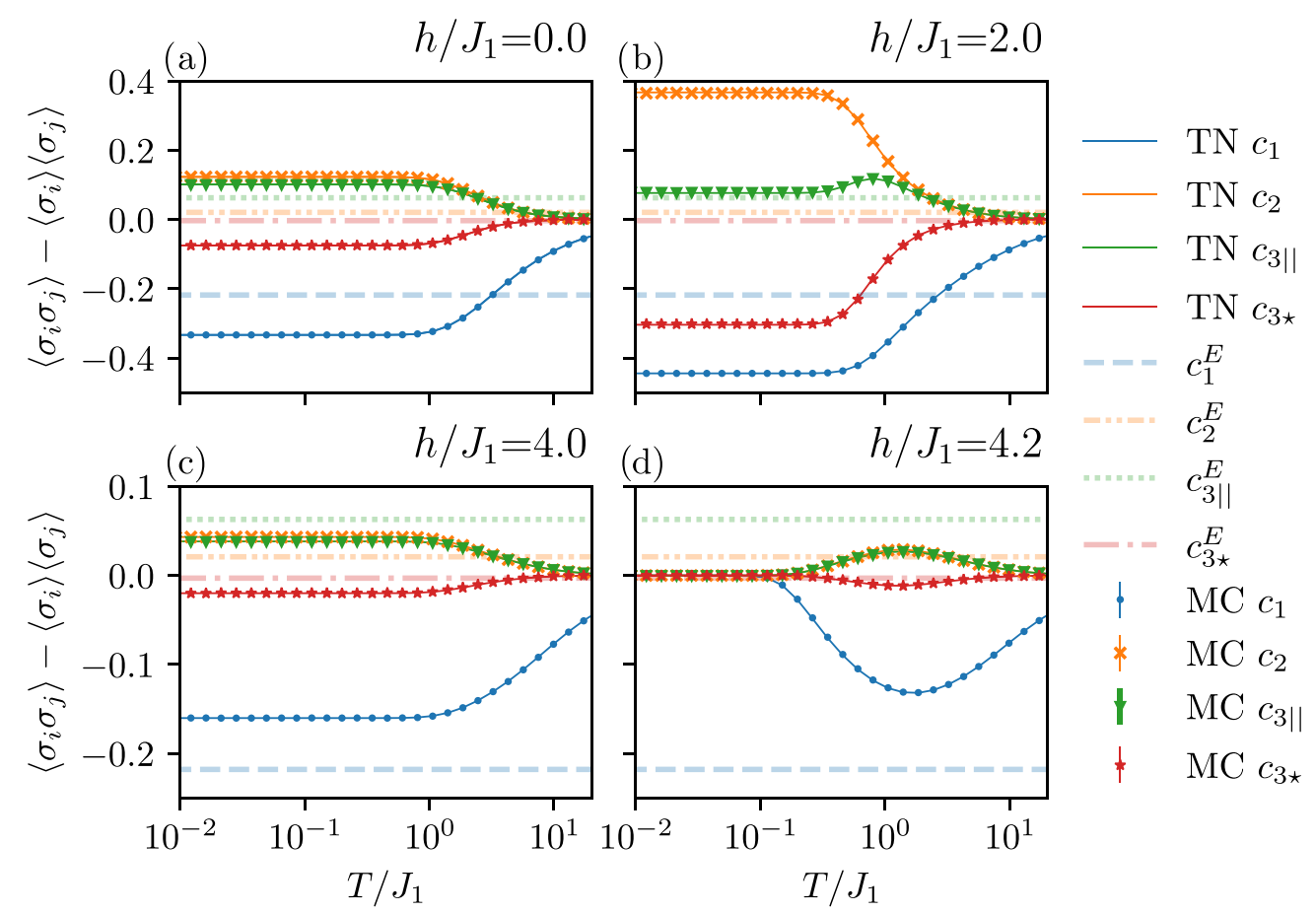

FIG. 11. Tensor networks (maximal bond dimension $\chi=80)$ and Monte Carlo $(N=2304$ sites) results for the first few correlations at specific magnetic fields. Note the difference in the vertical axis for subplots (a) $h=0$ and (b) $h=2 J_{1}$ versus (c) critical field $h=4 J_{1}$ and (d) $h=4.2 J_{1}$. See as well Tables IV and V for the values in the ground state. Notice that in the second row, we have zoomed in on the values of the correlations. The data for the correlations at regularly spaced fields can be found in Ref. [78].

change the descending order of the farther-neighbor correlations and explain the experimental observation that $c_{3 \|} \gtrsim 2 c_{2}$.

Here, we focus on the case with antiferromagnetic nextnearest-neighbor couplings for the Ising model. It should be noted that, upon changing the sign of the couplings and multiplying them by 2 , the model maps onto the spin-ice model on the kagome lattice with ferromagnetic next-nearestneighbor couplings [46,81]. We study the model with an $a d$ hoc Monte Carlo algorithm [68] and our dual tensor network construction from Fig. 6(b). The expression of the tensor on the triangle Eq. (20) remains unchanged, while Eq. (21) becomes

$$
H_{d_{1}, d_{2}, d_{3}, d_{4}, d_{5}, d_{6}}= \begin{cases}e^{-\beta J_{2}\left(\sum_{i=1}^{6} d_{i} d_{i+1}+2\right)} & \prod_{i} d_{i}=1 \\ 0 & \text { otherwise }\end{cases}
$$

where $d_{7}=d_{1}$.

The $J_{2}$ couplings form a set of three interpenetrating kagome sublattices. The two-up one-down, two-down one-up ice rule can be satisfied simultaneously on each triangle on the initial kagome lattice as well as on each triangle on these three kagome lattices. The ground-state energy per site is thus $[24,80]$

$$
E_{\mathrm{g} . \mathrm{s} .}=-\frac{2}{3} J_{1}-\frac{2}{3} J_{2} \text {. }
$$

Imposing these rules only leads to a partial lifting of the ground state degeneracy, and from Pauling estimates one gets a residual entropy per site [24] $S_{J_{1}, J_{2}} \cong \ln \left(2\left(\frac{3}{4}\right)^{(4 N / 3)}\right)=$ 0.3096. From the contraction of the tensor network, we get

$$
S_{J_{1}, J_{2}}=0.285299 \pm 1.4 \times 10^{-6}
$$

where the error is estimated from the difference between the value at maximal MPS bond dimension $(\chi=144)$ and the result of the fit in the infinite bond dimension limit. The result matches what we obtain with the method of Ref. [42] and our Monte Carlo thermodynamic integration result $S_{J_{1}, J_{2}} \cong$ $0.285 \pm 0.001$ (Appendices $\mathrm{C}$ and $\mathrm{E}$ ). In Table VI, we also give the first spin-spin correlations in the ground state, as obtained with both methods.

Takagi and Mekata [80] predicted a KT transition to this critical ground-state phase at temperatures of the order of $J_{2}$. Correspondingly, for small values of $J_{2}$, the specific heat exhibits two broad peaks corresponding to the two stages of the loss of entropy (first for imposing the ice rule on nearest-neighbor triangles and then for imposing it on fartherneighbor triangles), while for larger values of $J_{2}$, the two features merge into one $[46,80]$ (Appendix E). The behavior of the spin-spin correlations corresponds to this picture (Fig. 12), since we find that for small values of $J_{2}$ the correlations take their nearest-neighbor model value for a range of temperatures before going to their ground-state values, while

TABLE VI. First spin-spin correlations in the ground state of the next-nearest-neighbor model. For the Monte Carlo simulations, we show results with the number of sites $N=1296$ and at a temperature $T / J=7 \times 10^{-3}$.

\begin{tabular}{lcccc}
\hline \hline & $c_{1}$ & $c_{2}$ & $c_{3 \|}$ & $c_{3 \star}$ \\
\hline Dual TN $(\chi=144)$ & $-1 / 3$ & $-1 / 3$ & 0.5726 & 0.5933 \\
MC & $-1 / 3$ & $-1 / 3$ & $0.567 \pm 0.005$ & $0.586 \pm 0.005$ \\
\hline \hline
\end{tabular}




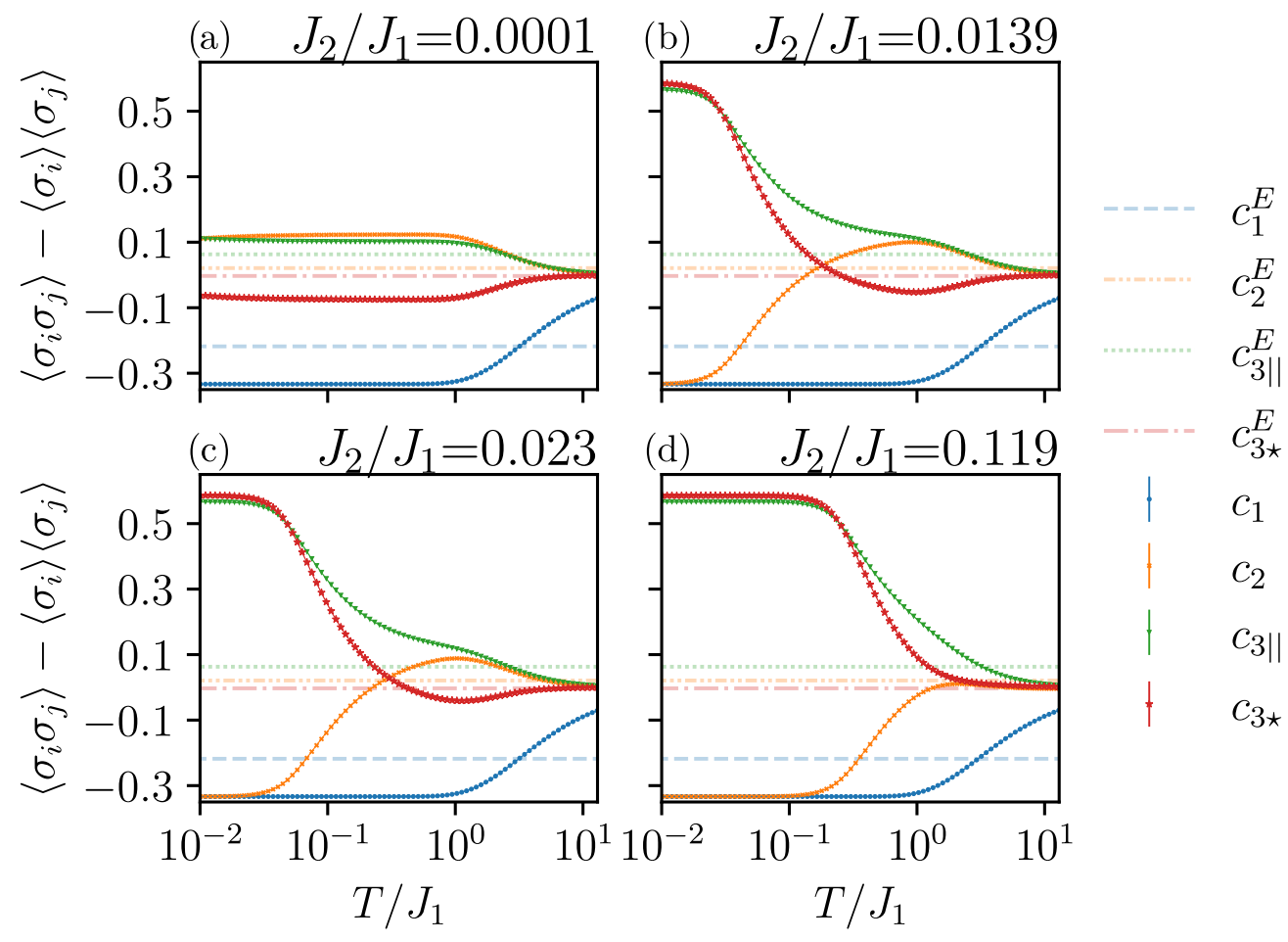

FIG. 12. Overview of the behavior of the first spin-spin correlations as a function of the temperature for various values of $J_{2}$, with Monte Carlo simulations for two system sizes $(N=576$ and 1296); the smaller system size is shown with a line while the larger one is shown with symbols. The dashed lines show the experimental values for comparison. The temperature at which the ground state correlations are reached depends on the value of $J_{2} / J_{1}$. (a) For small $J_{2}$, we recover the nearest-neighbor model correlations at intermediate temperatures. (b), (c) Upon increasing $J_{2}$, the region of temperature where $c_{2}>c_{3 \|}$ is pushed toward high temperatures and (d) eventually disappears. When $J_{2}$ is large, the $c_{2}$ correlations remain negative at any temperature. An overview is shown in Fig. 13.

for larger values of $J_{2}$ the competition between $J_{2}$ and $J_{1}$ significantly affects the correlations even at large temperatures. Note that Fig. 12 shows the values of the correlations with the Monte Carlo simulations for those ratios of $J_{2}$ to $J_{1}$ corresponding to the three scenarios discussed in Sec. II B $\left(J_{2}^{\text {dip }} / J_{1}, J_{2} / J_{1}, J_{2}^{\text {dip }} / J_{1}^{\text {dip }}\right)$.

Simulating the model systematically for a range of values of $J_{2}$, we can map out the values of the next-nearest-neighbor coupling and of the temperature where the spin-spin correlations are in a certain descending order. This is shown in Fig. 13, where one can see that-in agreement with the above discussion-for very small $J_{2} / J_{1} \lesssim 0.01$, there is a range of temperatures where the descending order of the correlations is compatible with the nearest-neighbor model. For $J_{2} / J_{1} \gtrsim 0.01$, one can see a broad region of temperatures and couplings where the spin-spin correlations are in the same relative order as in the experiment $\left(\left|c_{1}\right|>c_{3 \|}>c_{2} \gtrsim c_{3 \star}\right)$, and within this region, a non-negligible region where $c_{3 \|}>2 c_{2}$. In particular, for the micromagnetic value $J_{2}=0.023 J_{1}$, the temperature range where $c_{3 \|}>2 c_{2}$ is $0.3 \lesssim T / J_{1} \lesssim 0.6$. If $J_{2} \gtrsim 0.06 J_{1}$, the region where $c_{3 \|}>2 c_{2}$ extends all the way to $T=5 J_{1}$.

Although this model does not involve a magnetic field, it provides evidence that, to recover qualitatively the descending order of the spin-spin correlations, farther-neighbor couplings have to play a role. Additionally, it provides an idea of

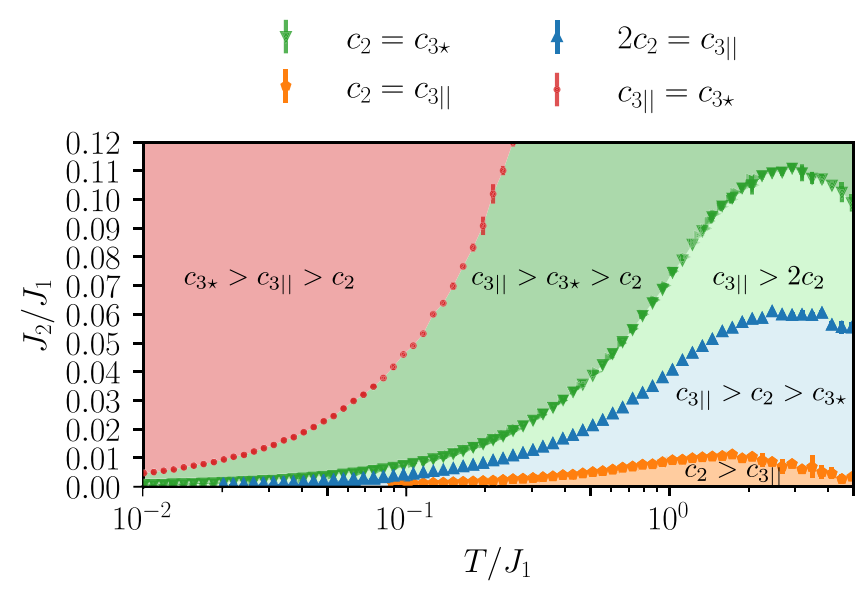

FIG. 13. Map of the descending order of the correlations as a function of the values of the temperature $T / J_{1}$ and the next-nearestneighbor coupling $J_{2} / J_{1}$ (obtained with MC simulations with $N=$ 576 sites). In the experiment, the correlations satisfy $c_{3 \|}>c_{2}>c_{3 \star}$, with $c_{3 \|} \gtrsim 2 c_{2}$ (light green region). For small enough $J_{2}$, there is a temperature region with $c_{2}>c_{3 \|}$ (orange region), compatible with the nearest-neighbor physics in terms of the descending order of the correlations. Importantly, the smallest value of $J_{2} / J_{1}$ in this graph is $J_{2} / J_{1}=0.001$ : for arbitrarily small $J_{2}$, the crossing $c_{2}=c_{3 \|}$ happens at arbitrarily small temperatures (Fig. 12). Note as well that at large temperatures, all the correlations are close to zero. 
how sensitive the correlations are to these farther-neighbor couplings.

\section{RESULTS IN THE $J_{1}-J_{2}-J_{3||}$ MODEL}

The micromagnetic simulations predict third-neighbor couplings that are almost half of the second-neighbor couplings. Since there is a competition between these couplings, we have to check that, in their presence, the predicted descending order of the correlations is still $c_{3 \|}>c_{2}>c_{3 \star}$. Additionally, we have seen in Sec. III that the proportion of triangles with a given magnetization can be accounted for by introducing a longitudinal magnetic field. We thus want to consider the following Hamiltonian:

$$
H=J_{1} \sum_{\langle i, j\rangle_{1}} \sigma_{i} \sigma_{j}+J_{2} \sum_{\langle i, j\rangle_{2}} \sigma_{i} \sigma_{j}+J_{3 \|} \sum_{\langle i, j\rangle_{3} \|} \sigma_{i} \sigma_{j}-h \sum_{i} \sigma_{i} .
$$

Because of the combination of farther-neighbor frustration and the presence of the field, this model is challenging to study using Monte Carlo simulations. At the same time, writing a converging tensor network formulation for such frustrated systems is far from trivial. Here, we present a study based on an understanding of the ground state using both methods, and results for the correlations as a function of the temperature using Monte Carlo simulations for small system sizes.

\section{A. Ground state of the $J_{1}-J_{2}-J_{3||}$ model with and without a field}

In zero field, the location of the phase boundaries and the value of the ground-state energy for the ground-state phase diagram has already been established [82], using exact ground-state lower bounds computed with Kanamori's method [84]. For antiferromagnetic couplings $J_{2}, J_{3 \|}>0$, there are four different ground-state phases. Our micromagnetic values for the couplings lie well within one of these phases, where

$$
E_{\mathrm{g} . \mathrm{s} .}=-\frac{2}{3} J_{1}-\frac{2}{3} J_{2}+\frac{2}{3} J_{3 \|} .
$$

As we will show, this phase exhibits a macroscopic ground state degeneracy.

In the ground state of frustrated systems, the standard formulation of tensor network partition functions based on Boltzmann weights typically fails $[42,85,86]$ - this is also the case for spin glasses. There are two possible approaches to this issue: one can either work around the problem of the multiplication of big and small numbers by adapting the tensor network algorithms to working with the logarithms of the Boltzmann weights [86] or one can use the insight that this failure is related to the presence of frustrated couplings and try to find the ground-state local rule to implement it at the level of the tensor, thereby relieving the frustration [42]. Here, we follow this second approach to construct a contractible tensor network that we can use to study the ground state.

In this farther-neighbor model, there is a special line for $J:=J_{2}=J_{3 \|}$, where the problem can be elegantly studied using a charge representation [31]. For positive $J$, this line coincides with two successive phase boundaries in the ground state of the $J_{1}-J_{2}-J_{3||}$ model. For $0<J<J_{1} / 3$, a classical
TABLE VII. Residual entropy in the ground state for $J_{2}=J_{3 \|} \lesssim$ 0.2 for the various ground-state phases. When indicated by the value of the field, we are looking at a phase boundary, and when indicated by the value of the magnetization plateau, we are looking at the phase between these boundaries.

\begin{tabular}{lcc}
\hline \hline & $\mathrm{TN}\left(\chi_{\max }=120\right)$ & Refs. [31,87] \\
\hline$h=0$ & $0.322273784 \pm 10^{-9}$ & 0.32 \\
$m=1 / 9$ & $0.125616 \pm 10^{-6}$ & $0.12(6)$ \\
$h=6 J_{3 \|}$ & $0.313746908 \pm 10^{-9}$ & - \\
$m=1 / 3$ & $0.107688 \pm 10^{-6}$ & $S_{\text {TLIAF }} / 3$ \\
$h=4\left(J_{1}+J_{2}\right)-6 J_{3 \|}$ & $0.26718206 \pm 10^{-8}$ & - \\
\hline \hline
\end{tabular}

spin liquid with an unusual residual entropy $S \cong 0.32$ (the hexamer classical spin liquid) was found in Ref. [31]. The effect of a longitudinal magnetic field on this phase has been recently studied in a follow-up work and was shown to give rise, in the ground state, to a number of magnetization plateaus with finite residual entropy [87]. We use these phases to check our tensor network construction in the presence of a magnetic field and find that the entropies we obtain for the various ground-state phases are in agreement with the existing results (Table VII).

As a first step in the tensor network construction, linear programming is used to build ground-state energy lower bounds based on splitting the Hamiltonian into terms defined on clusters $[42,88]$. We can thus determine the ground-state energy in the various phases by comparing the lower bound from this method and the upper bound from the Monte Carlo simulations; if they match, we have a proof for the value of the ground-state energy and we know that the tensor network will describe the complete ground-state manifold.

Our results for the ground-state phase diagram for $J_{3 \|}<$ $J_{2}$ are summarized in Fig. 14. In zero field, we recover the ground-state energy of Eq. (40), and from the tensor network construction, we find a residual entropy

$$
S_{J_{1}-J_{2}-J_{3 \|}}=0.143949 \pm 6 \times 10^{-6},
$$

which shows that some sort of classical spin liquid is available even when $J_{3 \|} \neq J_{2}$.

When introducing a finite magnetic field, the system enters an $m=1 / 9$ plateau which survives as long as $h<6 J_{3 \|}$. In this plateau, the ground-state energy is given by

$$
E_{1 / 9}=-\frac{2}{3} J_{1}-\frac{2}{3} J_{2}+\frac{2}{3} J_{3 \|}-\frac{1}{9} h
$$

We find a zero residual entropy in the thermodynamic limit by contracting the tensor network, but from the Monte Carlo simulations, we find that the ground states differ by nonlocal updates. In simulations with periodic boundary conditions, these updates correspond to strings of spins that cross the sample and close in on themselves through the periodic boundary conditions (Appendix G). If one looks at periodic boundary conditions by placing the lattice on a torus, then these updates are winding around the torus. Together with the tensor network result, this suggests that the ground-state degeneracy is macroscopic, but with a subextensive residual entropy, growing with the linear system size. 


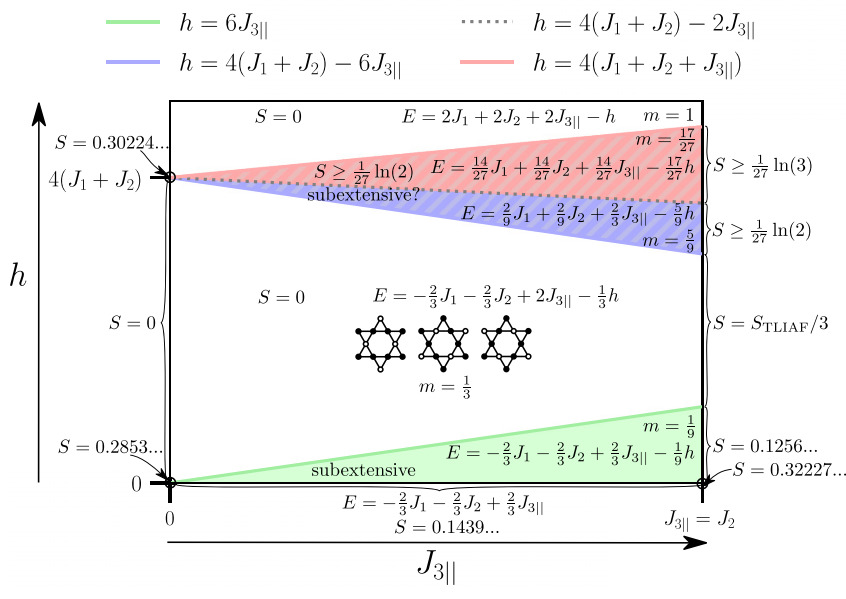

FIG. 14. Candidate ground-state phase diagram for the fartherneighbor model in a magnetic field at a fixed value of $J_{2}<\frac{1}{3} J_{1}$. The two hatched regions could only be studied with Monte Carlo computations, whereas the rest of the phase diagram was determined both with Monte Carlo simulations and with tensor networks. When the values are indicated next to arrows, they correspond to a specific point (for instance, we give the result for $J_{3 \|}=J_{2}$ and $h=0$ ) and when they are indicated next to brackets they correspond to a specific line (for instance, we give the results for $J_{3||}=J_{2}$ and various ranges of the field, which are different from the results for $J_{3 \|}<J_{2}$ ). The ground-state entropies are computed from tensor networks contractions, except for the two inequalities on the $J_{2}=J_{3 \|}$ line, studied in Ref. [87], and the lower bound in the $m=17 / 27$ plateau, estimated from the Monte Carlo results. The bottom left corner corresponds to the $J_{1}-J_{2}$ model. A magnetic field in this model immediately lifts the degeneracy to the long-range ordered stripe phase. For finite $J_{3 \|}$, there is a macroscopic ground-state degeneracy in zero field. With increasing field, there is first a magnetization plateau at $m=1 / 9$. Then, the long-range ordered stripe phase is selected, followed by a plateau at $m=5 / 9$ and another one at $m=17 / 27$ before saturation. For each phase, we give the ground-state energy, which is rigorously proved everywhere except in the two hatched regions.

The $m=1 / 3$ plateau corresponds to long-ranged ordered strings of nearest-neighbor up spins separated by down spins, with a ground-state energy

$$
E_{1 / 3}=-\frac{2}{3} J_{1}-\frac{2}{3} J_{2}+2 J_{3 \|}-\frac{1}{3} h .
$$

The phase boundary between the $m=1 / 9$ and $m=1 / 3$ plateau is thus found at $h=6 J_{3 \|}$. At this boundary, the ground states can have various magnetizations, corresponding to mixtures of states of both plateaus. Different sizes in the Monte Carlo simulations select different average magnetizations (Appendix $\mathrm{G}$ ); we therefore refrain from stating a value for the magnetization here.

The $m=1 / 3$ plateau extends until $h=4\left(J_{1}+J_{2}\right)-6 J_{3 \|}$, where we find a phase boundary. Above this field, the largest clusters that we used to split the Hamiltonian give an energy lower bound that does not match the exact ground-state energy [89]. However, we have evidence from Monte Carlo simulations (Fig. 15) that suggests the presence of $m=5 / 9$ and $m=17 / 27$ plateaus corresponding to the ones found at $J_{2}=J_{3 \| \mid}$ in Ref. [87]. The corresponding phase boundaries are at $h=4\left(J_{1}+J_{2}\right)-2 J_{3 \| \mid}$ and $h=4\left(J_{1}+J_{2}+J_{3 \| \mid}\right)$, which

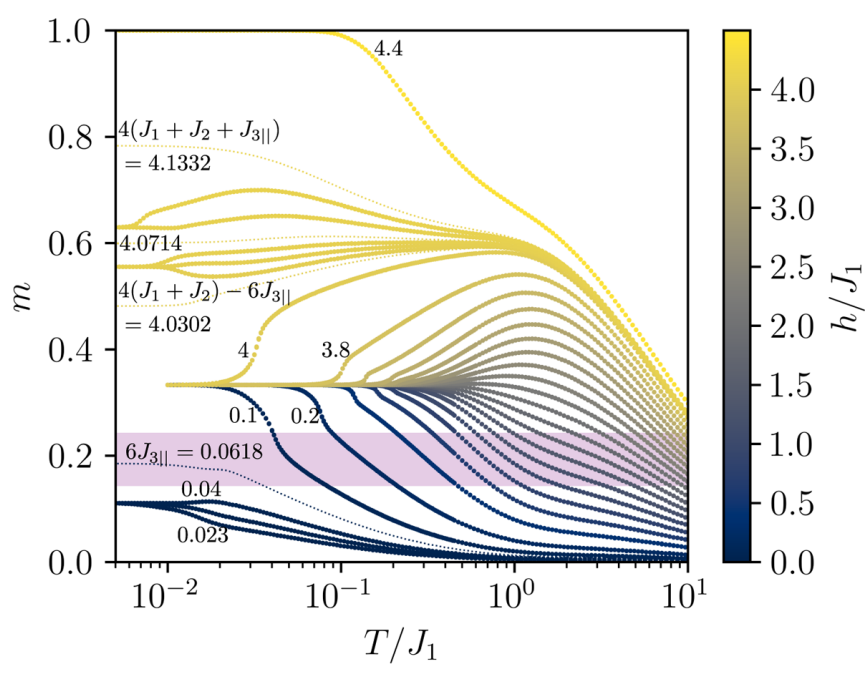

FIG. 15. Qualitative behavior of the magnetization as a function of the field and temperature in the $J_{1}-J_{2}-J_{3 \|}-h$ model. The points come from Monte Carlo simulations with $N=900$ in the $m=1 / 3$ plateau, with $N=144$ in the $m=5 / 9$ plateau and with $N=376$ in the other regions. The dotted lines are also from Monte Carlo simulations but correspond to the magnetization at the transition between plateaus; they are only given as a guide to the eye as the results strongly depend on the system size (Appendix G). The highlighted region corresponds to the region within one standard deviation of the experimental magnetization. The annotations indicate the value of the magnetic field for various curves.

means that this region is extremely reduced for our micromagnetic values of $J_{1}, J_{2}$, and $J_{3 \| \mid}$. In the $m=5 / 9$ plateau, with periodic boundary conditions in the Monte Carlo, we find again that ground states differ by strings of spins winding the torus, suggesting a subextensive residual entropy, whereas some local moves can be seen in the $m=17 / 27$ plateau, providing a lower bound for the residual entropy $S \geqslant \frac{1}{27} \ln (2)$ (Appendix G).

\section{B. Effect of the temperature and spin-spin correlations}

We finally give a qualitative discussion of the effects of temperature on the spin-spin correlation in this fartherneighbor model in a field and compare our predictions to the experimental results. In the rest of this section, we consider $J_{2}=0.023 J_{1}$ and $J_{3 \|}=0.0103 J_{1}$, corresponding to the micromagnetic simulations results. Figure 15 gives a qualitative picture of the magnetization as a function of field and temperature. Because the problem is a challenge for our Monte Carlo simulations (where we use the single-spin-flip algorithm combined with replicas in magnetic field and temperature), we only focus on small system sizes where needed. In Fig. 15, the selection of the various magnetization plateaus of the ground state with increasing fields is shown. With increasing temperatures, we find around the $m=1 / 3$ and $m=1 / 9$ plateau a behavior similar to the one in the $J_{1}-h$ model around the $m=1 / 3$ plateau (Appendix D): If the field is large, intermediate temperatures will give a larger average magnetization than the plateau value, whereas if the field is small, the magnetization immediately decreases with increasing temperatures. 


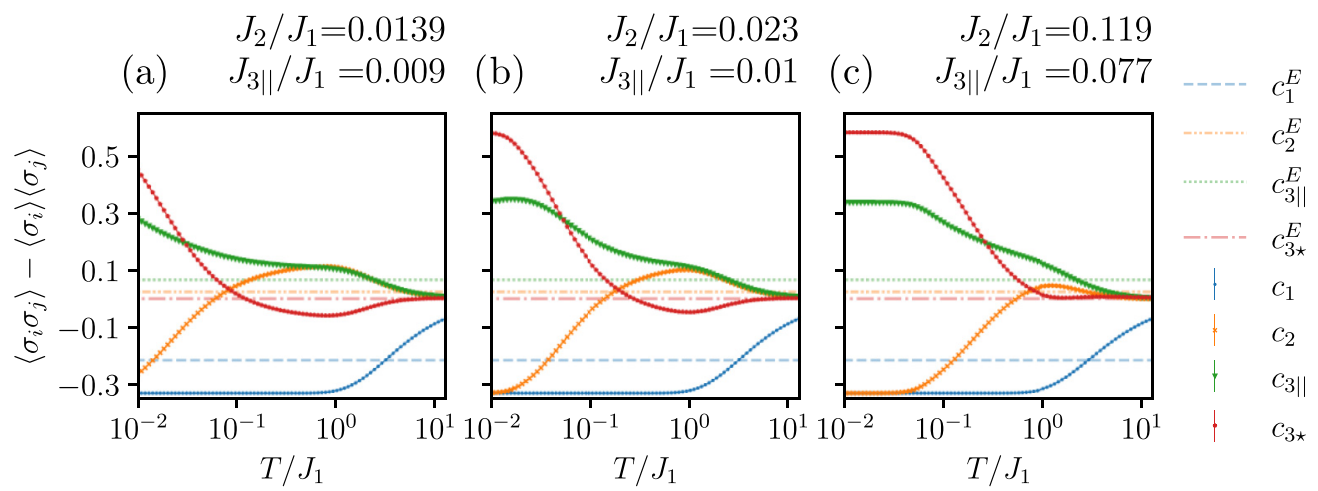

FIG. 16. Overview of the behavior of the first few correlations as a function of the temperature for the values of $J_{2}$ and $J_{3 \|}$ from micromagnetic simulations [(a) dipolar corrections to the nearest-neighbor model, (b) actual couplings with IP part, (c) purely dipolar model (no IP regions)], with Monte Carlo simulations for two system sizes $(N=576,1296)$. The smaller system size is shown with a line while the larger one is shown with symbols.

In analyzing the spin-spin correlations, we first consider Fig. 16, presenting the results in zero field. Although, in the ground state, the results are quite different from the $J_{1}-J_{2}$ model (Fig. 12), the qualitative result that there is a temperature range where $c_{3 \|} \gtrsim 2 c_{2}$ remains correct. This has to be contrasted to the nearest-neighbor model results, and shows that the experimental results can only be understood by taking into account farther-neighbor couplings, and that $J_{2}$ plays the important role, while $J_{3||}$ is simply not large enough to suppress the effect of $J_{2}$. It can also be seen in this figure that these results would be valid for a wide range of values of the couplings, including the dipolar case truncated to $3 \|$ neighbors.

Besides the descending order of the correlations, a qualitative characteristic of the experimental results is the presence of a nonzero magnetization, which we try to account for by introducing a magnetic field. We have seen that if there is a field in the experiment, we expect it to be very roughly of the order of $J_{2} \lesssim h \lesssim J_{1}$; in addition, when considering the percentages of triangles with a given magnetization, the nearest-neighbor model predicts $h \sim 1.6 J_{1}$. It thus seems sufficient to focus mainly on the two first magnetization plateaus. This is confirmed by the comparison between the experimen- tal magnetization and the Monte Carlo simulation prediction for the magnetization in Fig. 15. This result shows that it is sufficient to study the behavior in temperature for fields $6 J_{3 \|}<h<4\left(J_{1}+J_{2}\right)-6 J_{3 \| \mid}$ corresponding to the $m=1 / 3$ plateau. Figures 15,17 , and 18 give a consistent picture of the behavior of the model as a finite temperature is applied on the $m=1 / 3$ plateau ground state. At small fields, there is an intermediate temperature region where the system essentially behaves as the farther-neighbor model in zero field: the residual entropy of the nearest-neighbor model is recovered (Fig. 17) but the $c_{2}$ and $c_{3 \|}$ correlations are already inverted as compared to the nearest-neighbor model (Fig. 18). With increasing fields, the transition to the long-range ordered ground state (stripe phase) happens at higher temperatures, with stronger and stronger finite-size effects, reaches a maximum, then decreases again (Figs. 15 and 19). For $h=4 J_{1}$, at intermediate temperatures, we find approximately the residual entropy of the nearest-neighbor model (Fig. 17), with again an effect of the farther-neighbor couplings on the $c_{2}$ and $c_{3 \|}$ correlations (Fig. 15).

We want to verify that the zero field $c_{3 \|}>c_{2}$ region is preserved in the presence of a magnetic field. To compare to the experimental results, we show a map of the descending

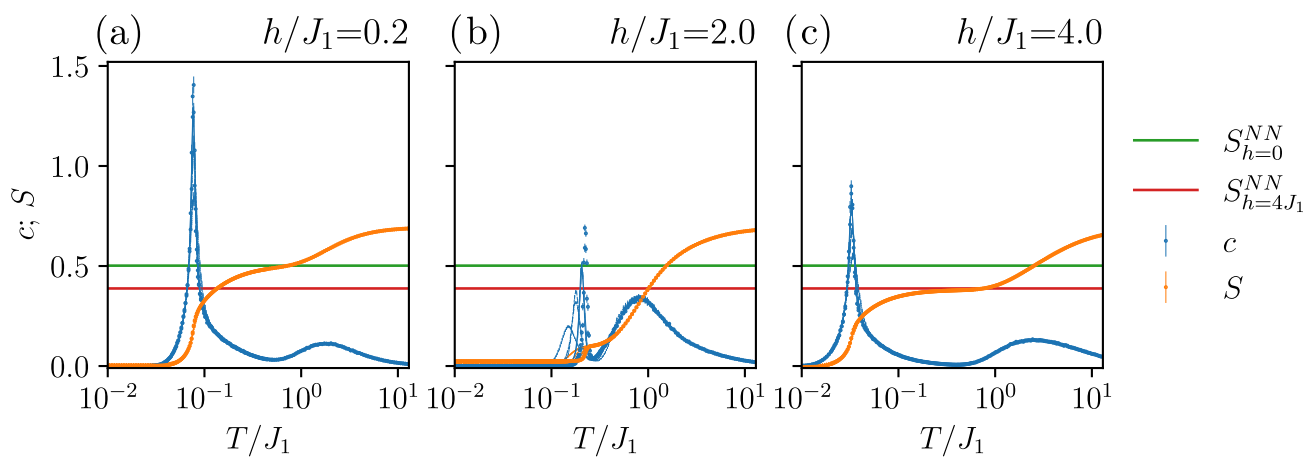

FIG. 17. Specific heat and residual entropy in the $m=1 / 3$ plateau for $N=36$ to $N=1296$ (markers). (a) At small fields, there is an intermediate temperature region where the entropy corresponds to that of the nearest-neighbor model in zero field, whereas (c) at large fields there is an intermediate temperature region where the entropy corresponds to the nearest-neighbor model in a field at $h=4 J_{1}$. (b) We note that at intermediate fields there are strong finite-size effects and the loss of residual entropy at the transition is not captured correctly by our simulations - the residual entropy in the ground state should be zero. 

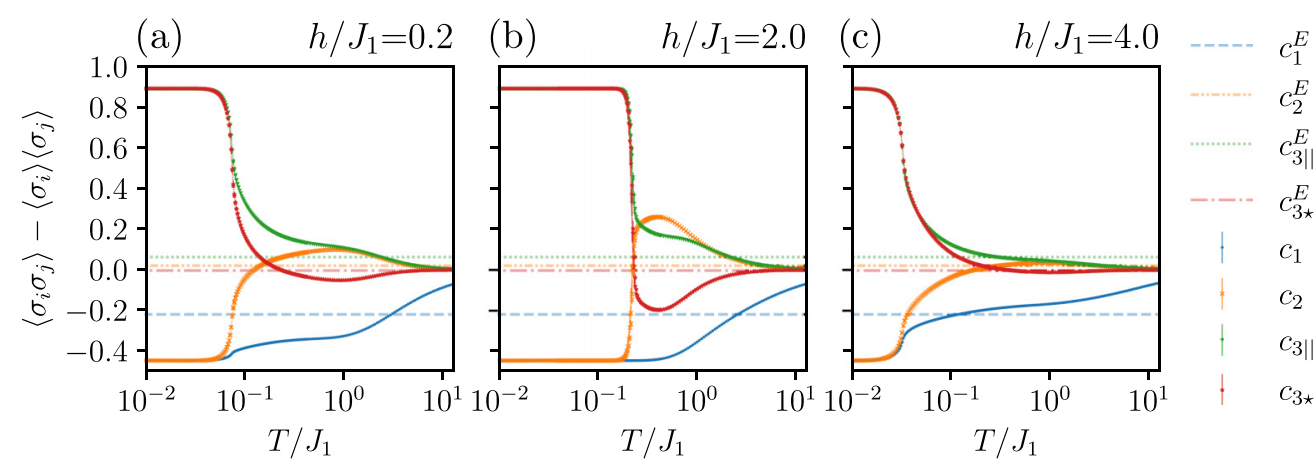

FIG. 18. Spin-spin correlations in the $m=1 / 3$ plateau for $N=900$ (dotted lines) and $N=1296$ (markers). The dashed lines indicate the experimental correlations, for comparison. (a) For small magnetic fields (region A in Fig. 19), there is an intermediate range of temperatures where the correlations correspond to the model in zero field (Fig. 16). (b) At intermediate field, the $c_{2}$ correlations again become larger than the $c_{3 \| \mid}$ correlations. (c) At large fields, there is an intermediate temperature region where the correlations are similar to those in the $J_{1}-h$ model at $h=4 J_{1}$, but with an effect of the farther-neighbor couplings which inverts the $c_{2}$ and $c_{3 \| \mid}$ correlations.

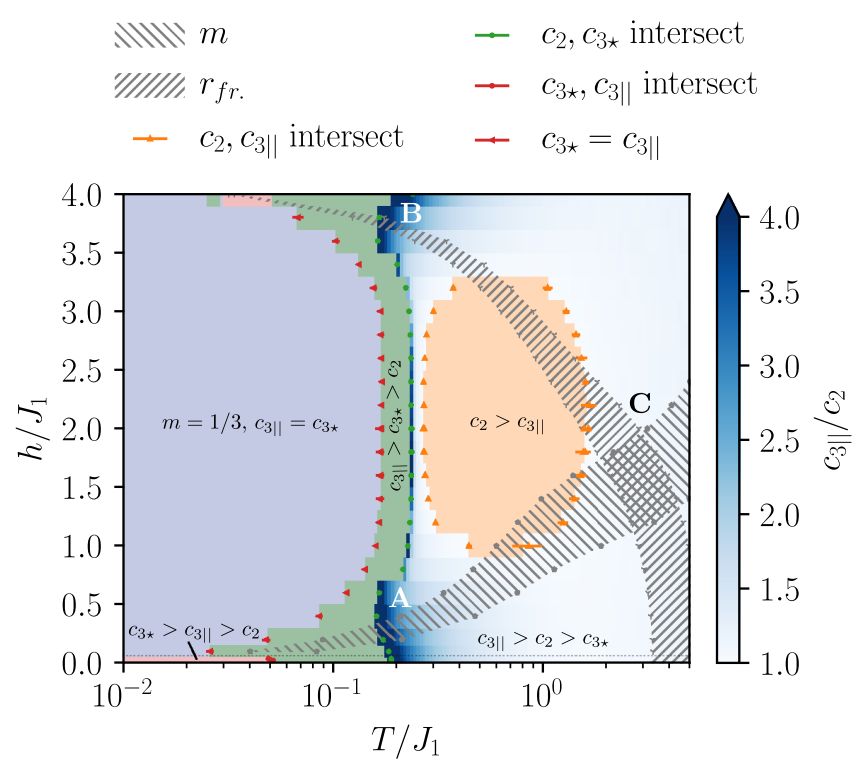

FIG. 19. Monte Carlo simulation results for the $J_{1}-J_{2}-J_{3 \|}-$ $h$ model as a function of the temperature. (A)-(C) The letters are placed just above three regions of interest, discussed in the main text. The various colored areas correspond to the values of temperature and fields where the correlations are in a certain descending order according to the Monte Carlo simulations. The pixels correspond to the 22 field points and 214 temperatures. With increasing temperature: in light blue, the region where the system is in the $m=1 / 3$ plateau ground state, with $c_{3 \|}=c_{3 \star}=8 / 9$; in light red, the region outside this plateau where $c_{3 \|}<c_{3 \star}$; in green, the region where the $c_{2}$ correlations are larger than $c_{1}$ but still smaller than $c_{3 \star}$; with the color map, the region where the descending order of the correlations is the same as in the experiment: $c_{3 \|} \geqslant c_{2}>c_{3 \star}$; in orange, the region where $c_{2}>c_{3 \|}>c_{3 \star}$. The hatched regions indicate the values of the temperature and field where the Monte Carlo simulations results for the magnetization $m$ and the proportion of ferromagnetic (frustrated) triangles $r_{f r}$. agree within the errors with the experimental values. The color map indicates the ratio of $c_{3 \| \mid}$ to $c_{2}$ in the region where the descending order of the correlations is the same as in the experiment; experimentally, this ratio is of the order of 2 to 3 in most samples (but $c_{2}$ can be negative in some samples, Fig. 5). order of the first four spin-spin correlations in Fig. 19, for $J_{2}=$ $0.023 J_{1}$ and $J_{3 \|}=0.0103 J_{1}$, as a function of the field and temperature. The region where the descending order of the correlations in the experiment $\left(c_{3 \|}>c_{2}>c_{3 \star}\right)$ is reproduced by the Monte Carlo simulations is shown by a color map, which gives the $c_{3 \|} / c_{2}$ ratio. For small fields $0 \leqslant h / J_{1} \lesssim 0.5$ and temperatures $0.1 \lesssim T / J_{1} \lesssim 1$, the $c_{3 \|}>c_{2}$ region indeed survives (with a reasonably large ratio of $c_{3 \|} / c_{2}$ ). At fields ranging from $0.8 J_{1}$ to $3.2 J_{1}, c_{2}$ becomes larger than $c_{3 \|}$ for $0.15 \leqslant T / J_{1} \leqslant 1.5$, which is consistent with the results of the nearest-neighbor model in a field, for which in this region the $c_{2}$ correlations are much larger than the $c_{3 \|}$ correlations. At large fields, $c_{2}$ decreases again such that $c_{3 \|}>c_{2}$ is valid for fields $3.5 \lesssim h / J_{1} \lesssim 4$. Thus, even in the presence of an external magnetic field in the farther-neighbor model, there are still regions that account for the order of spin-spin correlations in the experiment, with a reasonable ratio of $c_{3 \|}$ to $c_{2}$.

In Fig. 19, the hatched regions correspond to the values of field and temperature where the theoretical magnetization and the proportion of ferromagnetic triangles agree within the error bars with the experimental values. Although it demonstrates that it is not possible to account for all the experimental results at once, this graph highlights three regions of interest:

(1) Region $\mathbf{A}$ at low field $h / J_{1} \sim 0.2$ to 0.5 and temperatures $0.2 \lesssim T / J_{1} \lesssim 0.4$ where the Monte Carlo results for the magnetization match the experimental results and where the ratio of $c_{3||}$ to $c_{2}$ is similar to the experimental value, but where the Monte Carlo simulations predict zero ice-rule-breaking triangles. The corresponding correlation results are illustrated in Fig. 18(a) $\left(h=0.2 J_{1}\right)$; it can be seen that they are quite different from the experimental results.

(2) Region B at large field $3.4 \lesssim h / J_{1} \lesssim 3.8$ and intermediate temperatures $0.15 \lesssim T / J_{1} \lesssim 0.5$, where the combined effect of the temperature and magnetic field reproduces the experimental proportion of ice-rule-breaking triangles, and where the ratio of $c_{3 \|}$ to $c_{2}$ is again similar to the experimental value. However, in this region, the Monte Carlo simulations predict $\left\langle r_{m_{\Delta}=-1}\right\rangle=\left\langle r_{\left.m_{\Delta}\right\rangle=-3}=0\right.$ (all triangles are aligned with the field). Correspondingly, the magnetization predicted by the Monte Carlo simulations is around twice the experimental results $(0.35 \lesssim m \lesssim 0.5$ instead of $m \sim 0.2)$. The disconnected correlations for this region are shown in 


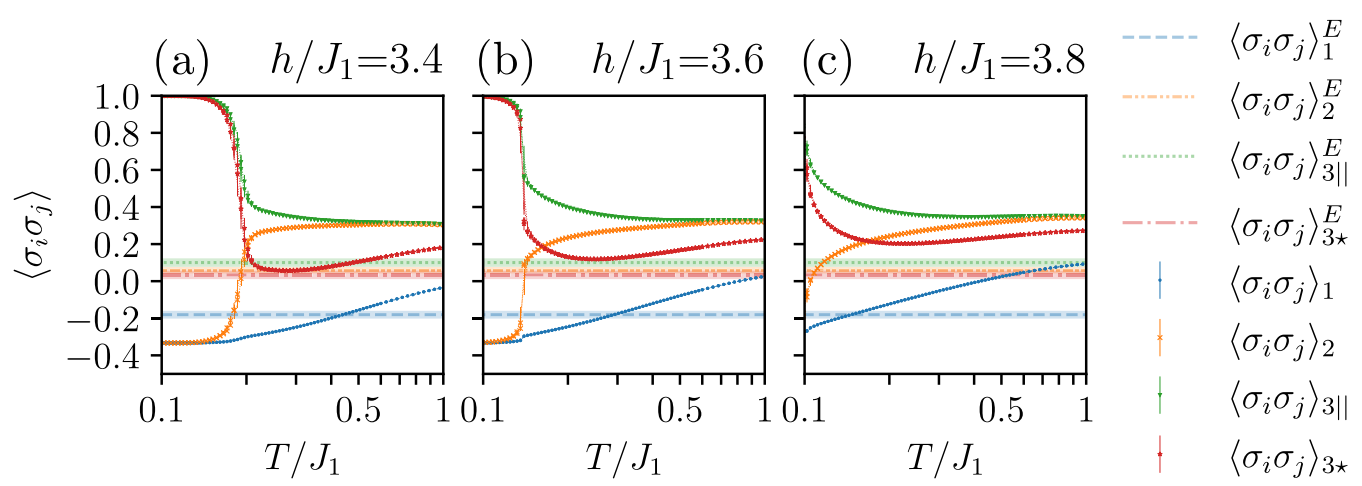

FIG. 20. (Region $\mathbf{B}$ in Fig. 19.) Behavior of the first few (disconnected) correlations at large magnetic field, in the window where $c_{3 \|} / c_{2}$ is of the same order of magnitude as in the experiment. The proportion of frustrated triangles is directly related to $\left\langle\sigma_{i} \sigma_{j}\right\rangle_{1}$; in this region, both are in agreement with the experimental value. Since the magnetization is very different from the experimental value, we plot the disconnected correlations (i.e., the correlations without removal of the squared magnetization) for a valid comparison.

Fig. 20; the farther-neighbor correlations are larger than their experimental counterpart.

(3) Region $\mathbf{C}$ at intermediate field $1 \lesssim h / J_{1} \lesssim 2$ and high temperatures $2 \lesssim T / J_{1} \lesssim 4$, where the number of triangles and the magnetization are both compatible with the experiment. Just as in the nearest-neighbor model in a field, requiring that all the percentages of triangles with given magnetization match with the experiment gives $h \cong 1.6 J_{1}$ [Fig. 21(a)]. However, Figs. 19 and 21(b) both show that, despite taking into account the farther-neighbor couplings, the correlations $c_{3 \| \mid}$ and $c_{2}$ remain essentially equal in this region.

\section{DISCUSSION}

From the analysis of the experimental data, we extract the main observation that $\left|c_{1}\right|>c_{3 \|}>c_{2} \gtrsim c_{3 \star}$. There are two additional qualitative observations: the systematic presence of a non-negligible proportion of ice-rule-breaking triangles and of a finite magnetization for all the samples, the magnetization of all the samples having the same sign. These two observations correspond to having precise proportions of triangles with magnetization $-3,-1,+1$, and +3 , given in Table II.

From the micromagnetic simulations, we obtain that the effect of the IP regions in the samples is first and foremost to increase the nearest-neighbor couplings significantly (approximately by a factor of 10), and second to slightly increase the second- and third-neighbor couplings, resulting in a system where the next-nearest-neighbor coupling $J_{2}$ is of the order of $2 \%$ of the nearest-neighbor coupling $J_{1}$, and the third-nearestneighbor coupling $J_{3||}$ is of the order of $1 \%$ of $J_{1}$.

Basing our analysis on the Monte Carlo and tensor networks simulations, we have asked the following questions:

(1) Considering an equilibrium distribution characterized by two Lagrange parameters - temperature and field-and corresponding to a model involving only nearest-neighbor couplings, can we reproduce the experimental proportions of triangles having a given magnetization?

(2) Considering this equilibrium distribution, is it possible to describe the descending order of the first spin-spin correlations in the experiment or does one have to consider a model involving farther-neighbor couplings? If so, is there a limit on how small these couplings have to be to become negligible?
(3) Finally, does the resulting equilibrium fully describe the experimental results, including the percentages of triangles with $m=+3,+1,-1,-3$, and the actual values of the correlations?
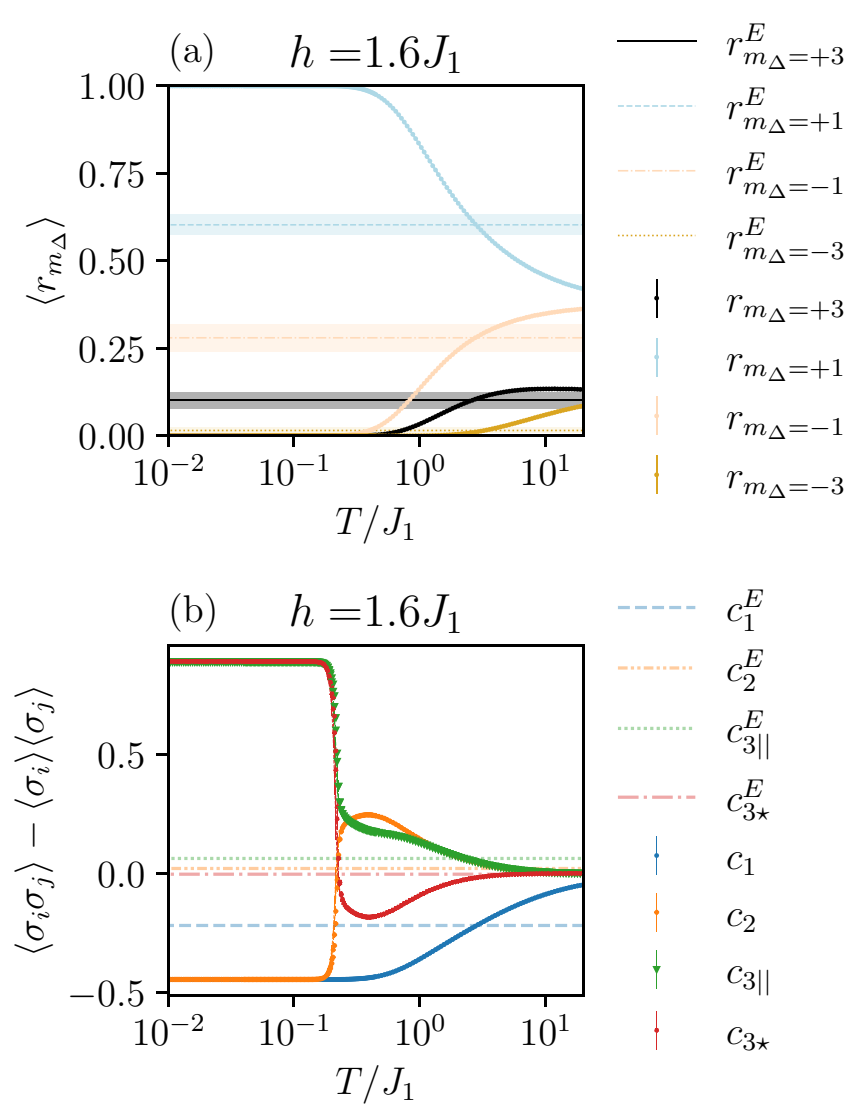

FIG. 21. (Region $\mathbf{C}$ in Fig. 19.) Monte Carlo results for two sizes $(N=900,1296)$. (a) Expectation value for the proportion of triangles with magnetization $m=-3,-1,+1,+3$ as a function of the temperature for an intermediate field. We recover the result of the nearest-neighbor model in a field (Fig. 10). (b) Behavior of the first few correlations at the same field. At intermediate temperatures, $c_{2}>c_{3 \|}$, and at large temperatures, $c_{2} \lesssim c_{3 \| \mid}$ (the two are essentially equal). 
The data in Fig. 10 allows us to easily determine that a simple model, the nearest-neighbor Ising antiferromagnet in a field, admits a region of field and temperature matching the experimental results for the number of triangles with a given magnetization. However, the corresponding temperature is quite large $\left(T \sim 2.8 J_{1}\right)$.

When taking into consideration the experimental spin-spin correlations and comparing them to the nearest-neighbor Ising antiferromagnet (Figs. 7 and 11), it is quite clear that the system is not accurately modeled by an equilibrium distribution if one simply neglects the farther-neighbor couplings altogether. Instead, the simulations of the $J_{1}-J_{2}$ model confirm that even a small next-nearest-neighbor coupling plays an essential role, affecting the correlations in a significant way (Figs. 12, 13, and 16). In the ground state, this is obviously the case because farther-neighbor couplings partially lift the macroscopic ground-state degeneracy of the nearest-neighbor model. What our results show is that, in addition, the correlations are also affected up to large temperatures, even for small values of $J_{2}$. For intermediate temperatures $\left(0.1 J_{1} \lesssim\right.$ $\left.T \lesssim 0.5 J_{1}\right)$, this modification can be sufficient to explain the descending order of the experimental correlations with respect to one another for $J_{2} \gtrsim 0.02$ (Fig. 12); for large temperatures ( $T \sim 3 J_{1}$ ), the next-nearest-neighbor couplings need to be larger $\left(J_{2} \gtrsim 0.06\right)$ to obtain the same result. The micromagnetic simulations predict a third-neighbor $\left(J_{3||}\right)$ coupling of the order of $J_{2} / 2$ (Fig. 4). This coupling naturally competes with the $J_{2}$ coupling; in the ground state, it further lifts the degeneracy, leading to a reduced residual entropy [Eq. (41)], and it affects the correlations (Fig. 14). At finite temperature, this third-neighbor coupling modifies the correlations as compared to the $J_{1}-J_{2}$ model, and therefore has to be taken into account. Despite this competition, a region with $c_{3 \|} \gtrsim 2 c_{2}$ is still present (Fig. 16).

In the farther-neighbor model, the magnetic field plays a similar role as in the nearest-neighbor model: Combined with the temperature, it selects the proportion of triangles with a certain magnetization, setting the overall magnetization (Fig. 15) and the number of frustrated triangles (Fig. 19). Additionally, it affects the correlations both in the ground state and at finite temperature. In the ground state, depending on the value of the field, the degeneracy gets either partially or completely lifted (Fig. 14) and, at experimentally relevant fields, the third-neighbor correlations can vary from $c_{3 \|}=$ $26 / 81(m=1 / 9$ plateau $)$ to $c_{3 \|}=8 / 9(m=1 / 3$ plateau $)$. At finite temperature, the region where $c_{3 \|}$ is larger than $2 c_{2}$ is preserved for two ranges of magnetic fields, illustrated by regions $\mathbf{A}$ and $\mathbf{B}$ in Fig. 19.

While the data in Fig. 19 demonstrates that the descending order of the correlations in the experiment can be recovered provided that farther-neighbor couplings are considered, this data also illustrates the impossibility of explaining simultaneously the entirety of the results based on these equilibrium distributions: the ratio of $c_{3 \|}$ to $c_{2}$ in most of the samples cannot be explained at the same time as the value of the magnetization and the proportion of ice-rule-breaking triangles. Based on the available information about the experiment, it is difficult to know in which farther direction to push the models. Nevertheless, three interesting regions can be observed, at intermediate temperatures for small field (region A) and large field (region B), and at large temperatures for intermediate fields (region $\mathbf{C}$ ).

In region $\mathbf{A}$, the magnetization matches the experimental results, but there is no ice-rule-breaking triangle, and correspondingly the nearest-neighbor correlations take their ground-state value, which is qualitatively very different from the experiment. To fully account for the experimental results, an additional mechanism would thus have to be invoked. Starting from a region with a smaller magnetic field than region $\mathbf{A}$, a possibility could be a source of disorder that would not be described by the temperature, such as a disorder in the coupling strengths generated by the IP regions or the presence of sites whose magnetization is not well defined, pinning ice-rule-breaking triangles at domain walls between low-energy states grown on the lattice, thus reducing the spinspin correlations and fixing the magnetization.

Region $\mathbf{B}$ is different, in that the percentage of ferromagnetic triangles, and correspondingly the expectation value $\left\langle\sigma_{i} \sigma_{j}\right\rangle_{1}$, correspond to the experimental results, but that the magnetization and the expectation values $\left\langle\sigma_{i} \sigma_{j}\right\rangle_{k}$ for the farther neighbors are larger than the experimental result. Again, in the absence of additional insight regarding the experiment, we can only suggest hypotheses involving another effect. In this case, it could perhaps be that, by switching domains of a certain size, the demagnetization protocol would preserve the number of ice-rule-breaking triangles, while reducing the farther-neighbor correlations and the magnetization.

Finally, region $\mathbf{C}$ is interesting because the experimental proportion of triangles with a given magnetization is well reproduced by the magnetic field and the temperature: no additional mechanism would be needed to describe the magnetization or the number of ice-rule-breaking triangles. However, the second- and third-neighbor correlations $c_{2}$ and $c_{3 \|}$ are essentially equal (when $J_{2}$ and $J_{3 \|}$ take the values predicted by the micromagnetic simulations), which does not seem to be in full agreement with the experiments. Looking at Fig. 13, one can see that in the $J_{1}-J_{2}$ model and for temperatures $T / J_{1} \sim 3$, the next-nearest-neighbor couplings should be of order $J_{2} \sim 0.06 J_{1}$ to $J_{2} \sim 0.11 J_{1}$ to reproduce the experimental correlations. This would correspond to at least a factor of 3 for $J_{2} / J_{1}$ as compared to the micromagnetic simulations. This seems unlikely, but we have to note that the micromagnetic simulations are performed for an idealized nanomagnet, and that the use of a square grid in the simulation did create a small difference between the nearest-neighbor couplings in two different directions $\left(J_{1, \mathrm{~h}}=\right.$ $1.868 \times 10^{-20} \mathrm{~J}$ while $\left.J_{1, \mathrm{~d}}=1.884 \times 10^{-20} \mathrm{~J}\right)$. However, the insight on the value of $J_{2}$ based on Fig. 13 needs to be taken into account very carefully as it would be affected by the presence of third-neighbor couplings and a magnetic field. We have not performed a systematic scan for a range of values of $J_{3 \|}$ vs $J_{2}$; we can therefore not conclude what range of couplings would provide a complete match with the experimental results in this region. Another difficulty with this region is conceptual: The corresponding effective temperature seems large and could be interpreted as an attempt to model disorder in the coupling strengths; in this context, it is difficult to decide how far a precise comparison between the predicted and experimental corre- 


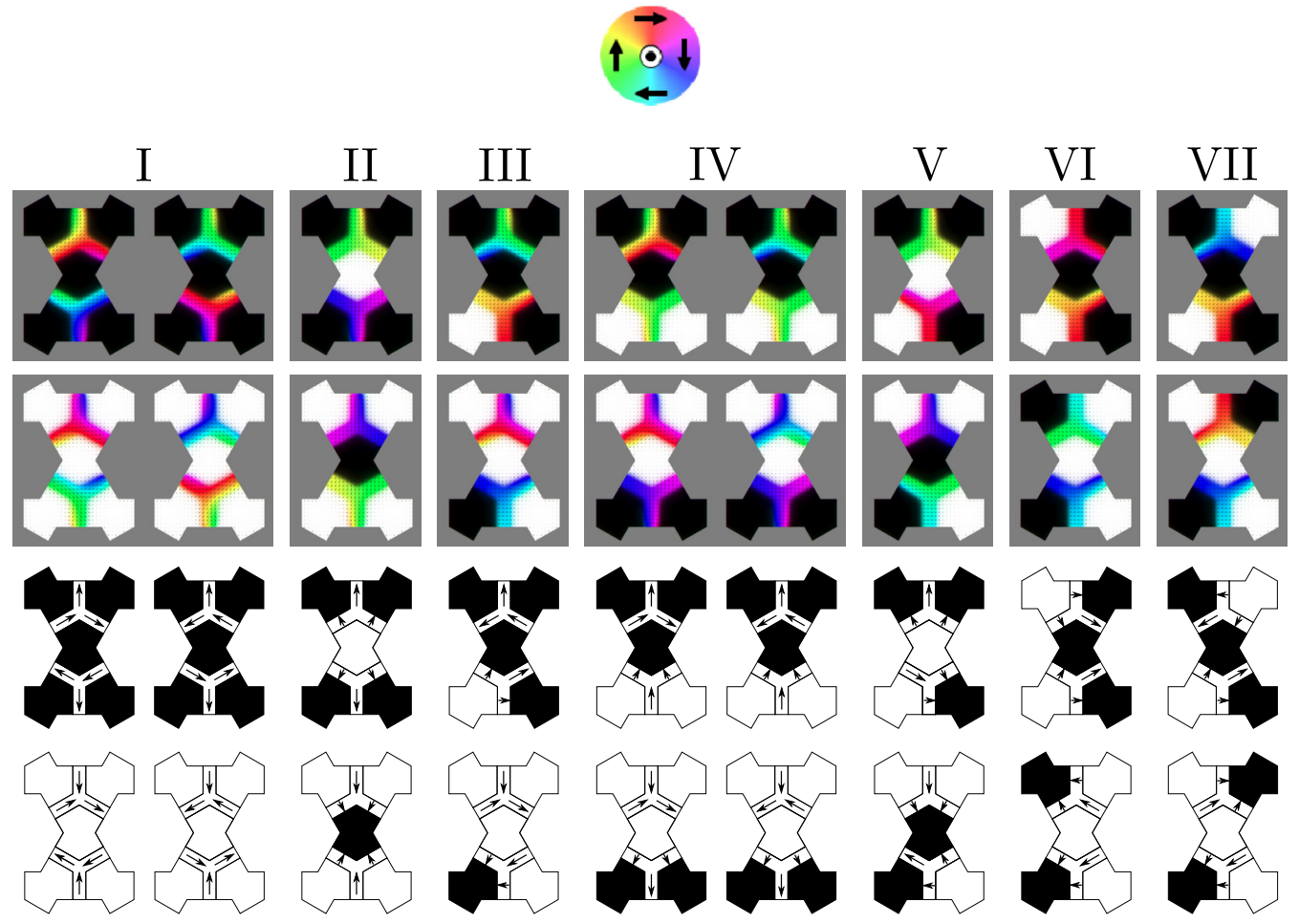

FIG. 22. First row: Micromagnetic simulations results (after relaxation) for each OOP configuration from Fig. 2. For each OOP configuration, we show the IP configuration that minimizes the energy. The colored disk at the top gives the legend for the IP magnetization. For the OOP regions, black means up Ising spin, white means down Ising spin. Second row: IP configurations that minimize the energy when all the spins are flipped. Here, we do not show the micromagnetic results for applying rotations and/or mirror symmetries, but they have been used in determining the average energy and the error bars for the results in Table VIII. Third and fourth rows: sketch illustrating the OOP and IP configurations from the first and second rows, for readability.

lations in this region can be pushed to draw meaningful conclusions.

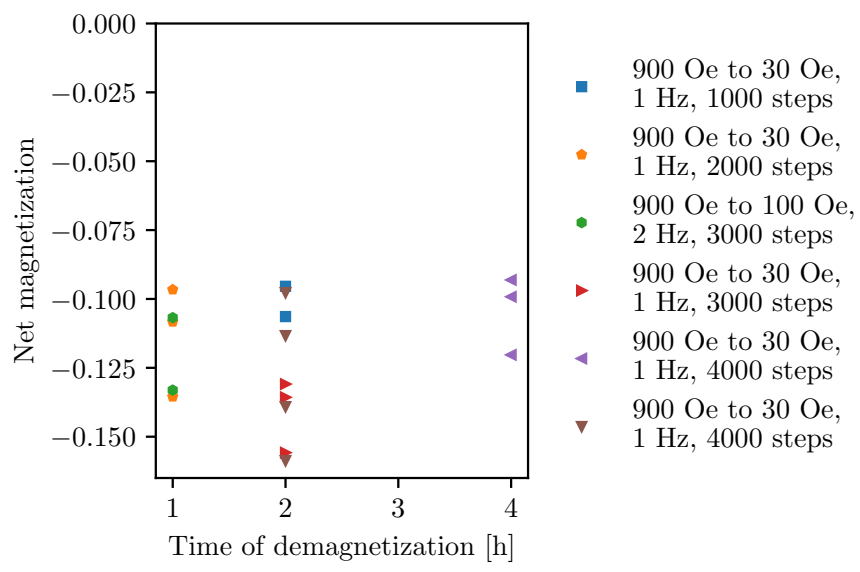

FIG. 23. MFM optimization of the demagnetization protocol, displaying various one-, two-, and four-hour protocols. Different colors at each protocol time indicate different protocols and each point represents an individual array whose net magnetization is computed. The net magnetization cannot be driven closer to zero than -0.09 . The more extended protocols do not show a clear trend toward reducing the net magnetization. The larger spread at two hours is due to more samples being tested. To be efficient in measuring our 20 arrays, the two-hour protocol was chosen.

\section{SUMMARY AND OUTLOOK}

In this paper, we have presented Monte Carlo and tensor network results for a series of short-range antiferromagnetic Ising models on the kagome lattice, in the presence of a longitudinal field, computing the first few spin-spin correlations systematically. These models are combined to give
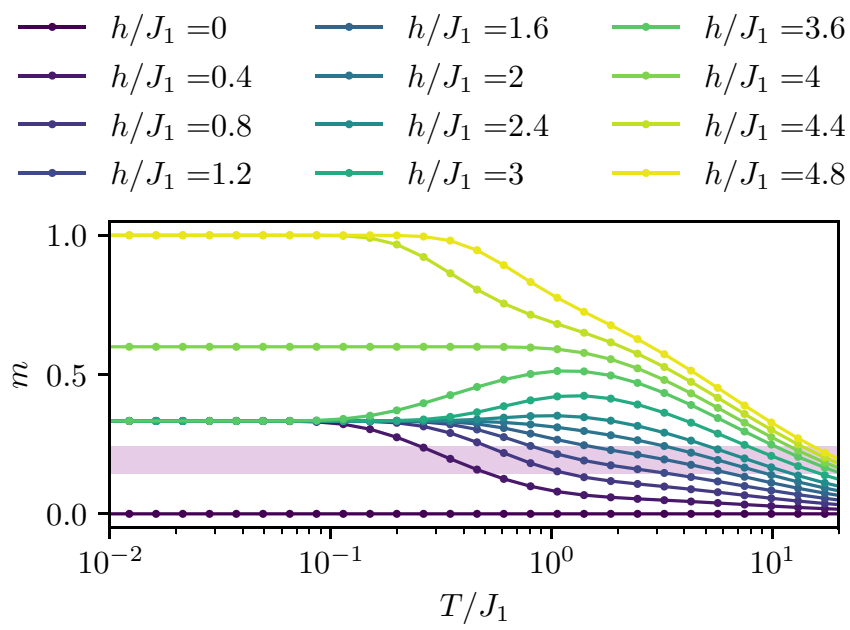

FIG. 24. magnetization as a function of the field and the temperature for the nearest-neighbor Ising antiferromagnet in a magnetic field. 
TABLE VIII. Results of the micromagnetic simulations for the energies of the various configurations from Fig. 2, for an IP width of $50 \mathrm{~nm}$.

\begin{tabular}{lcrr}
\hline \hline & Expression of the energy & Energy (pure OOP) $\left[10^{-17} \mathrm{~J}\right]$ & Energy $(\mathrm{OOP}+\mathrm{IP})\left[10^{-17} \mathrm{~J}\right]$ \\
\hline I & $E_{0}+4 J_{1, \mathrm{~d}}+2 J_{1, \mathrm{~h}}+2 J_{2}+2 J_{3 \|}+Q_{1}+4 Q_{2}$ & $-1.645897 \pm 6 \times 10^{-7}$ & $-1.2408601 \pm 5 \times 10^{-7}$ \\
II & $E_{0}-4 J_{1, \mathrm{~d}}+2 J_{1, \mathrm{~h}}+2 J_{2}+2 J_{3 \|}+Q_{1}-4 Q_{2}$ & $-1.6609718 \pm 2 \times 10^{-7}$ & $-1.367404 \pm 1 \times 10^{-6}$ \\
III & $E_{0}+2 J_{1, \mathrm{~d}}-Q_{1}-2 Q_{2}$ & $-1.65413964 \pm 8 \times 10^{-7}$ & $-1.3064207 \pm 4 \times 10^{-7}$ \\
IV & $E_{0}+2 J_{1, \mathrm{~h}}-2 J_{2}-2 J_{3 \|}+Q_{1}$ & $-1.6549028 \pm 2 \times 10^{-7}$ & $-1.3062638 \pm 4 \times 10^{-7}$ \\
V & $E_{0}-2 J_{1, \mathrm{~d}}-Q_{1}+2 Q_{2}$ & $-1.6616731 \pm 7 \times 10^{-7}$ & $-1.3693708 \pm 5 \times 10^{-7}$ \\
VI & $E_{0}-2 J_{1, \mathrm{~h}}+2 J_{2}-2 J_{3 \|}+Q_{1}$ & $-1.661483 \pm 2 \times 10^{-7}$ & $-1.3699101 \pm 6 \times 10^{-7}$ \\
VII & $E_{0}-2 J_{1, \mathrm{~h}}-2 J_{2}+2 J_{3 \|}+Q_{1}$ & $-1.661797 \pm 9 \times 10^{-7}$ & $-1.3707522 \pm 5 \times 10^{-7}$ \\
\hline \hline
\end{tabular}

the $J_{1}-J_{2}-J_{3 \|}-h$ model, for which we established a candidate ground-state phase diagram as well as the temperature dependence of the magnetization and first correlations. In light of these simulations together with micromagnetic computations, we have studied the experimental results obtained from an array of chirally coupled nanomagnets on the kagome lattice.

The micromagnetic simulations show that arrays of chirally coupled nanomagnets using DMI are a good basis for investigating models with extremely strong nearest-neighbor antiferromagnetic couplings. Indeed, even though short-range farther-neighbor couplings are strengthened as well, the micromagnetic simulations suggest that they increase by a much smaller factor, resulting in an effective model which is significantly different from the dipolar Ising case.

Our Monte Carlo and tensor network simulations show that even very small farther-neighbor couplings significantly affect the first few spin-spin correlations, and that the next-nearestneighbor coupling $J_{2}$ cannot be neglected even if it is as small as $2 \%$ of the nearest-neighbor coupling $J_{1}$. Additionally, our results show how the correlations are affected by the introduction of a third-neighbor coupling $J_{3 \|}$ and a magnetic field $h$. Their effect on the spin-spin correlations is summarized in Figs. 14 (for the ground state) and 19 (for the descending order of the correlations as a function of the temperature).

Although Fig. 19 shows that it is not possible to obtain a complete quantitative description of all the results of the experiment, it allows us to spot two regions of interest (A and $\mathbf{B})$ at small and at large fields which seem to contain the essence of the experimental results (namely, the descending order of the correlations) and might serve as a support to explain the observations modulo the introduction of an additional ingredient (a source of defects at weak field and a mechanism of reduction of the magnetization at large field). A third region (C) shows that a combination of an intermediate field and a surprisingly large temperature (suggesting the presence of disorder in the coupling strength due to local changes in the DMI or in the IP width, or disorder corresponding to the presence of nanomagnets whose local magnetization is not well determined from our measurements) can reproduce the experimental proportions of triangles with a given magnetization but not the difference between the second- and third-neighbor correlations.

We hope that our results can motivate and support further research both on the experimental and theoretical side. Experimentally, our contribution is to make a step in the direction of tuning couplings for frustrated Ising models on the kagome lattice. Realizing a similar system but where the IP part separating three nearest-neighbor sites on the kagome lattice would have a triangular hole might change the effective model. A priori, one could expect that the nearest-neighbor couplings would remain large but that the effective secondand third-neighbor couplings would be smaller [90]. This is, however, a technical challenge because of the small size of the triangle that needs to be created. It is also not clear whether this could help to recover the nearest-neighbor correlations, since $J_{2}$ should still be of the order of $1 \%$ of $J_{1}$; as Figs. 12 and 16 show, the results will also depend on the ratio of $J_{3||}$ to $J_{2}$. Another possible direction of improvement would be to have measurements allowing the determination of the configuration of the IP regions as well as the OOP regions [91] to confirm the micromagnetic simulations predictions and check that the
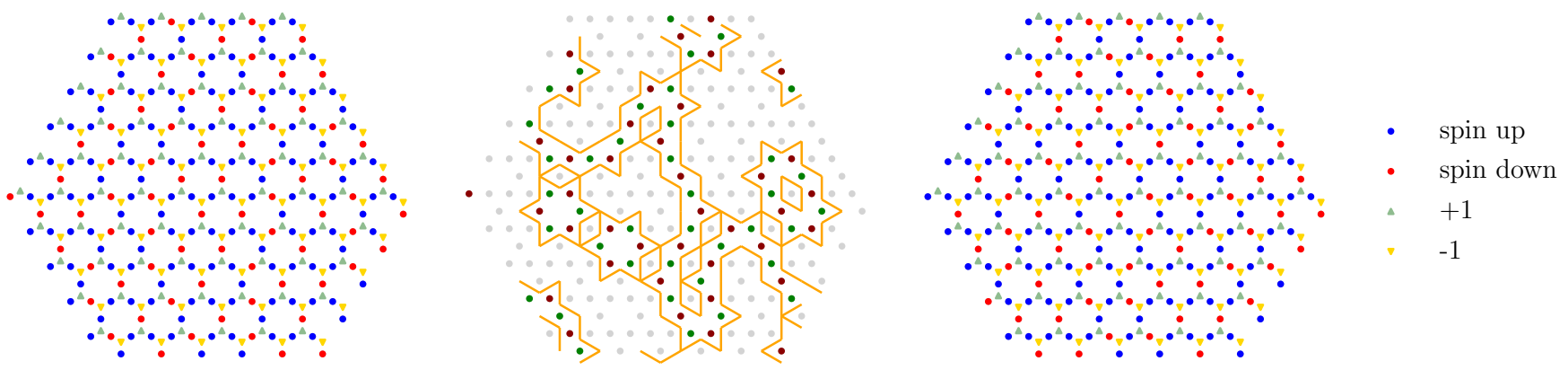

FIG. 25. Example of two ground-state configurations in the nearest-neighbor model in a magnetic field $0<h<4 J_{1}$ (leftmost and rightmost panels) and the difference between the two configurations (central panel). The orange lines delimit the regions where spins are flipped. They correspond to updates in the classical dimer configuration on the (dual) dice lattice. The green dots correspond to spins that are flipped from up to down, and the dark red dots to spins that a flipped from down to up. Comparing the two ground states, it can be seen that the underlying charge configuration is long-range ordered, while the spin configuration is not. 

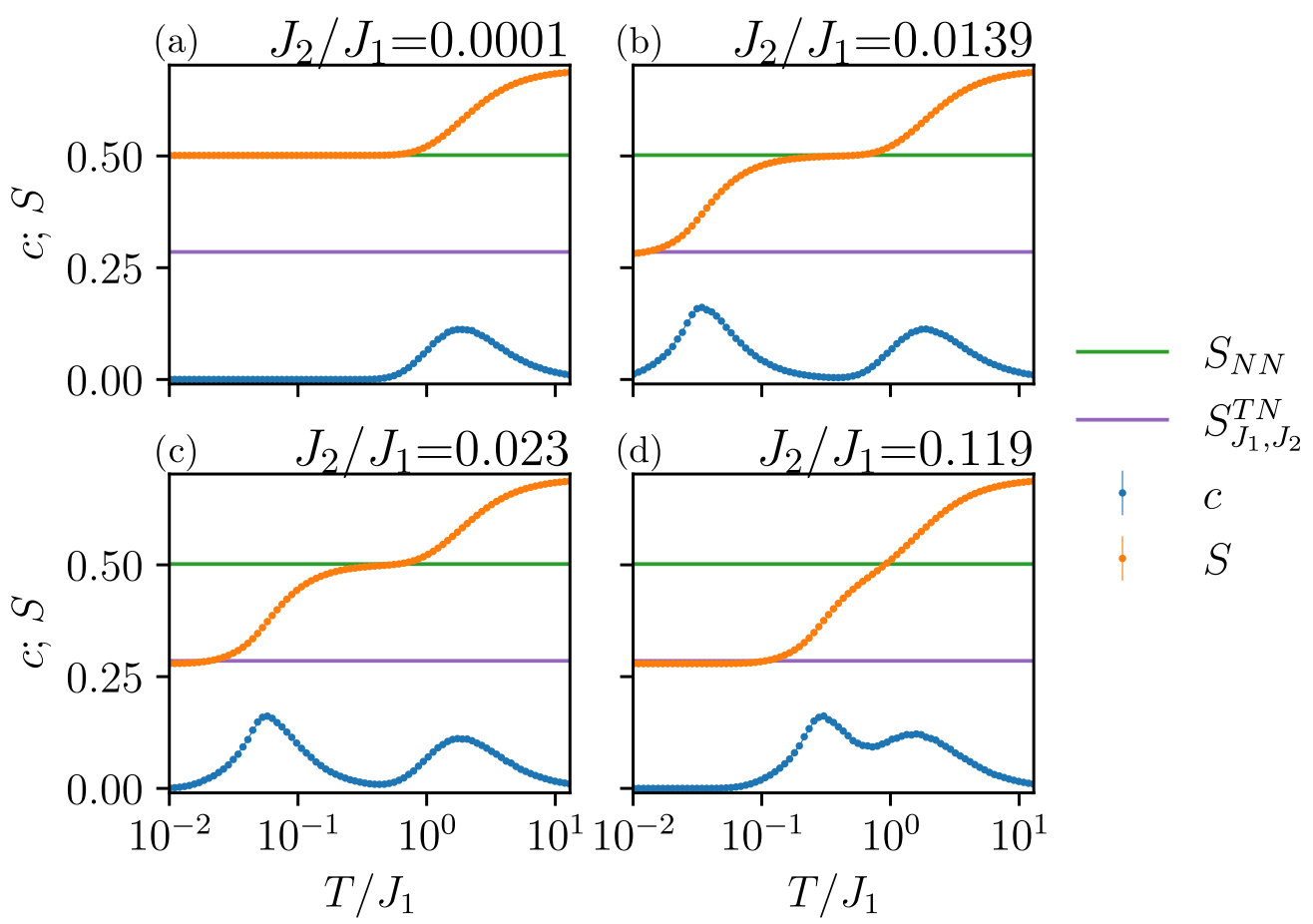

C $S$

FIG. 26. Overview of the behavior of the specific heat and entropy as a function of the temperature for various values of $J_{2}$, with Monte Carlo simulations for two system sizes $(N=576,1296)$. The smaller system size is shown with a line while the larger system size is shown with symbols.

IP regions do not get stuck in configurations which do not minimize the energy.

On the theoretical side, we have uncovered the phase diagram of the $J_{1}>J_{2}>J_{3 \| \mid}$ model in a field, exhibiting an interesting range of macroscopically degenerate phases, some with subextensive residual entropy, hopefully motivating further investigation. Besides the Hamiltonian considered here, we see two main directions for more involved models. The first is to determine whether the observed effects could be due to disorder in the coupling strengths or the presence of vacant sites by modeling the effect that such disorder would have on the spin-spin correlations. The second, assuming absence of disorder in coupling strength, would be to perform micromag- netic simulations on larger structures to determine the impact that the IP regions have on effective long-range couplings in this system and, in particular, to see if these long-range couplings are different from the well-studied dipolar model. Comparing the values of these longer-range couplings to the predicted short-range four-site couplings (Fig. 2) seems to be an essential step to build a more accurate model, including the next order of interactions.

Data availability. The experimental data, which served as a basis for this project, as well as the micromagnetic, Monte Carlo, and tensor network data produced for this paper, are available on Zenodo [78]. The Monte Carlo code for this paper is available upon reasonable request to J.C.

$J_{2} / J_{1}=0.0139$
(a) $J_{3 \mu} / J_{1}=0.009$

$J_{2} / J_{1}=0.023$
$J_{3||} / J_{1}=0.01$

$J_{2} / J_{1}=0.119$
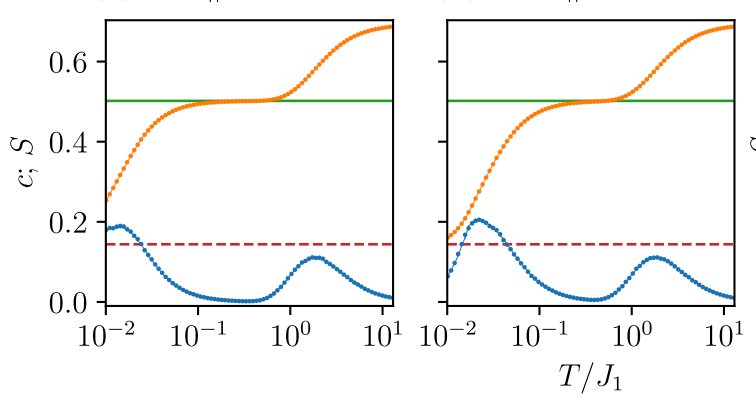

(c) $J_{3 \|} / J_{1}=0.077$

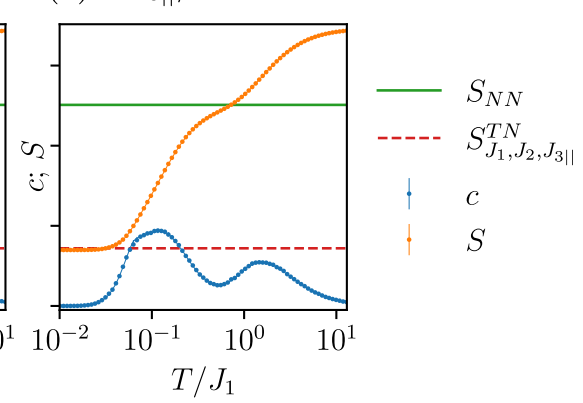

FIG. 27. Overview of the behavior of the specific heat and entropy as a function of the temperature for the values of $J_{2}$ and $J_{3 \|}$ from Sec. II B, with Monte Carlo simulations for three system sizes $(N=576,1296)$. The smaller system size is shown with a line while the larger is shown with symbols. 


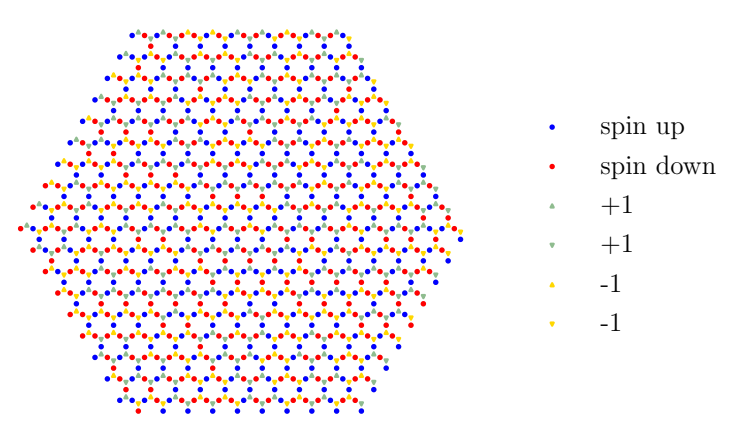

FIG. 28. An example of a ground-state configuration for the $J_{1}-$ $J_{2}-J_{3||}$ model, including the corresponding charge configuration.

\section{ACKNOWLEDGMENTS}

J.C. and F.M. thank the QUTE group at Ghent University for giving them access to their VUMPS implementation and, in particular, Frank Verstraete, Laurens Vanderstraeten, and Bram Vanhecke for a number of fruitful discussions on tensor networks and frustration. They are also grateful to Andrew Smerald for relevant discussions on the $J_{1}-J_{2}$ model and Monte Carlo simulations. J.C. also thanks Natalia Chepiga, Jonathan D'Emidio, Mithilesh Nayak, and Patrick Emonts for interesting blackboard discussions. J.C. and F.M. are supported by the Swiss National Science Foundation (Project No. 200020_182179). L.J.H. and K.H. acknowledge financial support from the Swiss National Science Foundation (Project No. 200020_172774). X.W. acknowledges the ThinkSwiss Research Scholarship. A.H. was funded by the European Union's Horizon 2020 research and innovation program under Marie Skłodowska-Curie Grant Agreement No. 794207 (ASIQS). Part of the computations have been performed using the facilities of the Scientific IT and Application Support Center of EPFL (SCITAS).

J.C. is the corresponding author for the Monte Carlo and tensor network data, K.H. for the experimental data, and both J.C. and K.H. for the micromagnetic simulations data and analysis.

\section{APPENDIX A: DEMAGNETIZATION PROTOCOL}

Several demagnetization protocols with different parameters were tested using the net magnetization of a specific array on a sample as a measure of success. However, the spread in the net magnetization for different samples was larger than any improvement in the magnetization achieved with the protocols when going from a one-hour protocol to four hours (Fig. 23), and a zero net magnetization was not reached. This is different from results for dipolar coupled nanomagnets, where it has been found advantageous to use longer protocols [92], as is demonstrated, for instance, by Ref. [93]. While we did not observe such a clear trend in our demagnetization protocols, we should note that our samples and protocols are significantly different from the usual dipolar coupled nanomagnets as our samples have chirally interacting IP and OOP regions. In addition, we do not rotate the sample in the demagnetizing field. In our chirally coupled systems, rotating the sample would induce strong interactions between external fields and the IP regions.

\section{APPENDIX B: MICROMAGNETIC SIMULATIONS RESULTS}

The aim is to estimate the values of the various coupling constants relative to the full nearest-neighbor coupling, to determine if they are large enough to explain the inversion of the $c_{2}$ and $c_{3 \|}$ correlations observed in Fig. 5 as compared to the nearest-neighbor models in Figs. 7 and 11. To do this, we simulate the various configurations of Fig. 2 for the Ising spins and let the system relax. We then compare their respective energies to extract the effective couplings. For the configurations of Fig. 2, indexed by roman numbers, we obtain the micromagnetic results shown in Fig. 22. In Table VIII, we summarize the expression of the energy (based on Fig. 2) and the value of the energy in the micromagnetic simulations, first in the pure OOP case (dipolar couplings, no IP region), and second in the complete case shown in Fig. 22. The errors in the energies are estimated from the differences in the energies of configurations that belong to the same group.

\section{APPENDIX C: MONTE CARLO SIMULATIONS}

The Monte Carlo simulations algorithms depend on the problems we are studying. In zero field, we use the algorithm of Rakala and Damle [68], which consists of mapping spin configurations to dimer configurations on the dual (dice) lattice and building loop updates in this dual space which respect local detailed balance and can thus be accepted once they close. In the presence of a field, the dual updates are typically rejected, and we resort to a simpler single-spin-flip update. This is, however, not sufficient, as those updates are typically rejected at low temperature. To alleviate the problem, we use replicas in field and in temperature (known as parallel tempering in the case of the replicas in temperature) $[73,79,94]$. When we cannot compare to the tensor networks results, the simulations are always completed for a number of sizes until convergence, and for at least two independent runs for each size. The correlations are always computed as an average over the sample, and then an ensemble average over the set of measurements. The errors are estimated from a binning analysis [95]. Each Monte Carlo step (MCS) consists of $\left(N_{\text {spin }}, N_{\text {worm }}, N_{\text {rep }}\right)$ single spin flip, worm, and replica updates. The parameters for the various figures are as follows:

(1) Figure $7 \quad\left(8 N_{\text {sites }}, 8 N_{\text {sites }}, 1\right)$ : thermalization-1024 MCS, measurements - 16384 spaced by three MCS.

(2) Figure $11\left(8 N_{\text {sites }}, 8 N_{\text {sites }}, 1\right)$ : thermalization-1024 MCS, measurements-16 384 spaced by three MCS.

(3) Figures 12 and $26\left(4 N_{\text {sites }}, 4 N_{\text {sites }}, 1\right)$ : thermalization4096 MCS, measurements-32 768 spaced by eight MCS.

(4) Figure 13 : thermalization-4096 MCS, measurements-8192 spaced by eight MCS.

(5) Figure $15 \quad\left(4 N_{\text {sites }}, 0,1\right)$ : thermalization-262 144 MCS, measurements - 16384 spaced by 16 MCS (except for the $1 / 3$ plateau, see parameters for Figs. 18 and 17).

(6) Figures 16 and $27\left(4 N_{\text {sites }}, 4 N_{\text {sites }}, 1\right)$ : thermalization -16384 MCS, measurements-32 768 spaced by eight MCS.

(7) Figures 17, 18, and 20: $\left(4 N_{\text {sites }}, 0,1\right)$ : thermalization65536 MCS, measurements_-8192 spaced by eight MCS. 

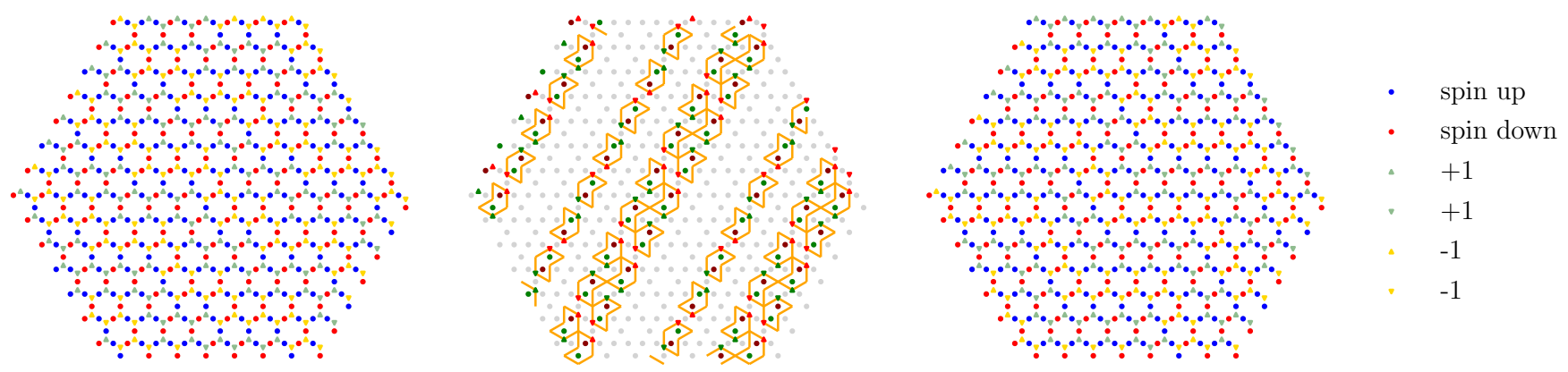

FIG. 29. Two examples of ground-state configurations for the $J_{1}-J_{2}-J_{3 \|}$ model, including the corresponding charge configuration, for a magnetic field $0<h<6 J_{3||}(m=1 / 9)$. The central panel shows the difference between the two configurations, with the following convention: The orange lines delimit the regions where spins are flipped, the green (dark red) dots correspond to spins that are flipped from up to down (down to up), and the red (green) triangles correspond to charges that are changed by $+2(-2)$.

(8) Figure $21\left(4 N_{\text {sites }}, 0,1\right)$ : thermalization-65 $536 \mathrm{MCS}$, measurements - 8192 spaced by eight MCS.

The residual entropy is computed from a thermodynamic integration of the energy [96]. At inverse temperature $\beta$, one has

$$
S(\beta)=\ln (2)+\beta E(\beta)-\int_{0}^{\beta} E\left(\beta^{\prime}\right) d \beta^{\prime},
$$

where here $E$ stands for the energy per site. Because in practice the integration is only done up to $\beta_{0}=1 / T_{\max }$, the residual entropy at high temperature is estimated analytically based on the high-temperature expansion of the specific heat:

$$
c_{\text {High } T}(\beta) \cong \beta^{2}\left(2 J_{1}^{2}+2 J_{2}^{2}+2 J_{3||}^{2}+h^{2}\right) .
$$

\section{APPENDIX D: MORE ABOUT THE $J_{1}-h$ MODEL}

In Fig. 24, we give an overview of the magnetization as a function of the field and the temperature for the nearestneighbor Ising antiferromagnet on the kagome lattice. The highlighted region corresponds to the magnetization within one standard deviation of the experimental value. The results are obtained from systematic tensor network contractions as described in Sec. III. For each field and temperature, we

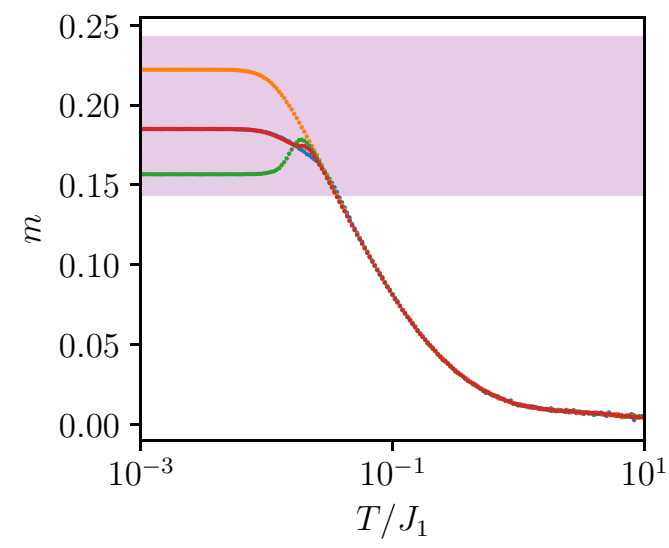

$L=3$

$L=4$

$L=5$

$L=6$

FIG. 30. Magnetization as a function of the temperature for the $J_{1}-J_{2}-J_{3 \|}$ model at $h=6 J_{3 \|}$ for various small system sizes. The number of sites is $N=9 L^{2}$. The shaded region corresponds to the experimental value. also computed the first spin-spin correlations and found that $c_{2} \geqslant c_{3 \|}$ everywhere.

In Fig. 25, Monte Carlo ground-state configurations of the nearest neighbor in a field for the $m=1 / 3$ plateau are illustrated. For these configurations, it can be easily verified that the underlying charge configuration of the nearest-neighbor model in the $1 / 3$ plateau is long-range ordered (corresponding to the two-up one-down rule).

\section{APPENDIX E: MORE ABOUT THE $J_{1}-J_{2}$ MODEL}

In Fig. 26, the specific heat and the entropy per site as a function of the temperature for two different system sizes in the $J_{1}-J_{2}$ model are shown. The selected values of $J_{2}$ correspond to the panels of Fig. 12. As expected, we find two features in the specific heat- two regimes where a loss of entropy takes place. The feature at larger temperatures, which does not change from one panel to the next, corresponds to the nearest-neighbor model, as can be seen from the fact that it leads to an entropy of $S \cong 0.502$. The second feature corresponds to the selection, within the ground state of the nearest-neighbor model, of those configurations that respect an ice rule for the $J_{2}$ kagome sublattices.

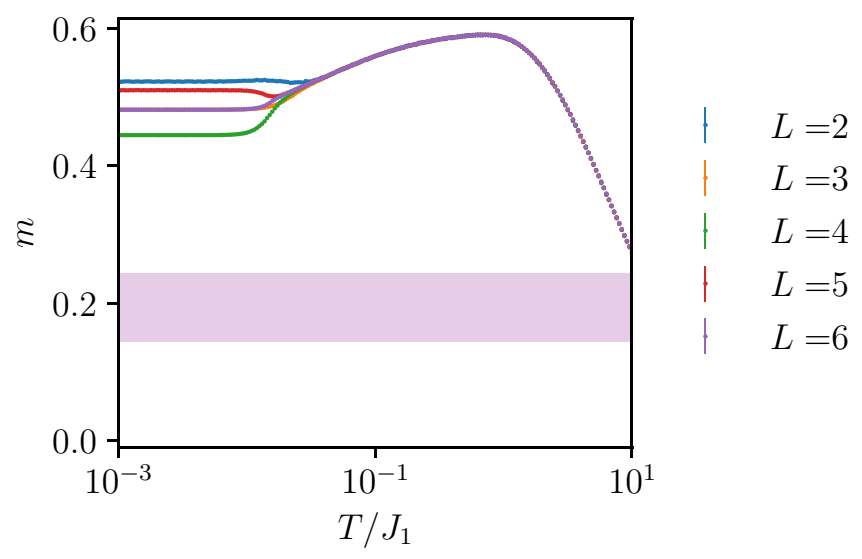

FIG. 31. Magnetization as a function of the temperature for the $J_{1}-J_{2}-J_{3||}$ model at $h=4\left(J_{1}+J_{2}\right)-6 J_{3||}$, for various small system sizes. The shaded region corresponds to the experimental value. 

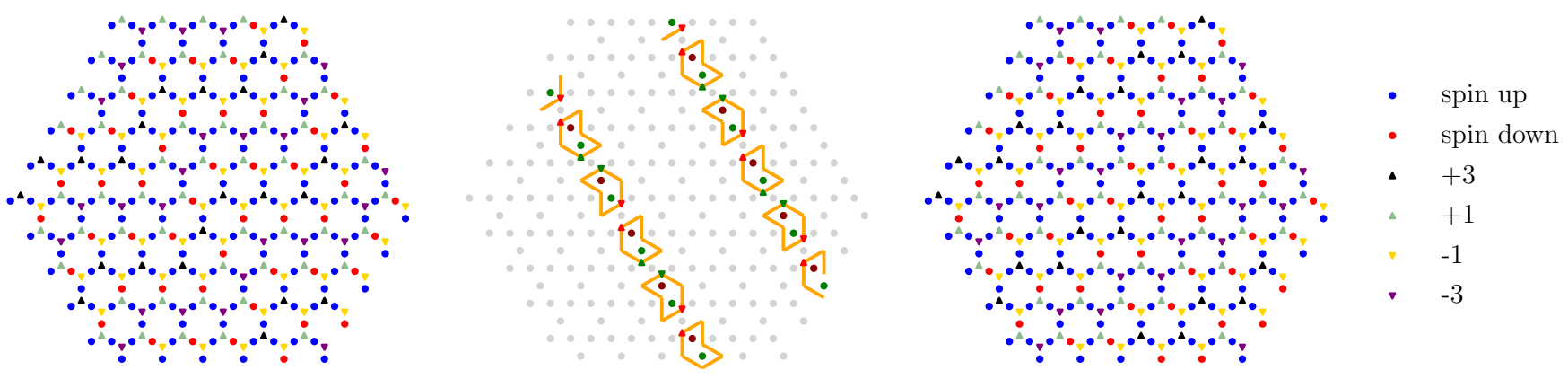

FIG. 32. Two examples of ground-state configurations for the $J_{1}-J_{2}-J_{3 \|}$ model, including the corresponding charge configuration, for a magnetic field $4\left(J_{1}+J_{2}\right)-6 J_{3 \|}<h<4\left(J_{1}+J_{2}\right)-2 J_{3 \| \mid}(m=5 / 9)$. The central panel shows the difference between the two configurations (see the caption of Fig. 29 for the convention regarding the updates).

\section{APPENDIX F: MORE ABOUT THE $J_{1}-J_{2}-J_{3||}$ MODEL IN ZERO FIELD}

In Fig. 27, we show the specific heat and the entropy per site as a function of the temperature for two different system sizes in the $J_{1}-J_{2}-J_{3 \|}$ model in zero field. The selected values of $J_{2}$ and $J_{3 \|}$ correspond to the panels of Fig. 16, i.e., to the values that have been discussed in the micromagnetic simulations section. Again, we find two peaks in the specific heat. Exactly as in the $J_{1}-J_{2}$ case, the feature at larger temperatures corresponds to the nearest-neighbor model. The second feature is actually a combined effect of the $J_{2}$ and $J_{3 \|}$ couplings. The $J_{2}$ ice rule is imposed but, additionally, the $c_{3 \|}$ correlations are restricted to the minimum that they can reach in the $J_{1}-J_{2}$ set of ground states. An example ground state is shown in Fig. 28.

\section{APPENDIX G: DETAIL OF THE $J_{1}-J_{2}-J_{3||}-h$ GROUND STATES}

As discussed in the main text, the phase diagram of the farther-neighbor model in a field arises from a selection of a different set of states depending on the value of the magnetic field. In zero field, tensor network results, as well as Monte Carlo results, show that there is a residual entropy even for $0<J_{3 \|}<J_{2}$. Upon introducing the magnetic field, this macroscopic ground-state degeneracy is immediately partially lifted. Two examples of such ground states are shown in Fig. 29. The difference between the two configurations illustrates the type of torus-winding updates that can be found (i.e., nonlocal updates that cross the sample and close in on themselves through the periodic boundary conditions). Each such update can be performed independently. Together with the fact that the tensor network contraction gives zero residual entropy, this provides evidence that the ground-state degeneracy is macroscopic but grows exponentially with the linear system size.

Upon increasing the magnetic field, there is a first-order phase transition in the ground state between the $m=1 / 9$ and $m=1 / 3$ plateaus. There, the states are a mixture of the $m=1 / 9$ ground states and the stripe state of the $1 / 3$ plateau, and the average magnetization shows a strong dependence in system size (only sizes with a linear size of a multiple of 3 match), Fig. 30.

In the $m=1 / 3$ plateau, the charge state is the same as in the corresponding plateau of the nearest-neighbor model, but the farther-neighbor couplings select a long-range ordered stripe state (as long as $J_{3 \|}<J_{2}$; for $J_{3 \| \mid}>J_{2}$ another longrange ordered state is selected). At the transition to the $m=$ $5 / 9$ plateau, we again find a strong size dependence of the magnetization (Fig. 31). The $m=5 / 9$ plateau seems again characterized by a subextensive ground-state degeneracy, with nonlocal updates winding the torus (Fig. 32); however, we do not have tensor network results in this phase. Finally, in the $m=17 / 27$ plateau, we find that there are local updates, shown in Fig. 33, yielding a lower bound for the residual entropy in that phase, $S \geqslant \frac{1}{27} \ln (2)$.

The present discussion is a preliminary study of this ground-state phase diagram and much is left to be clarified.
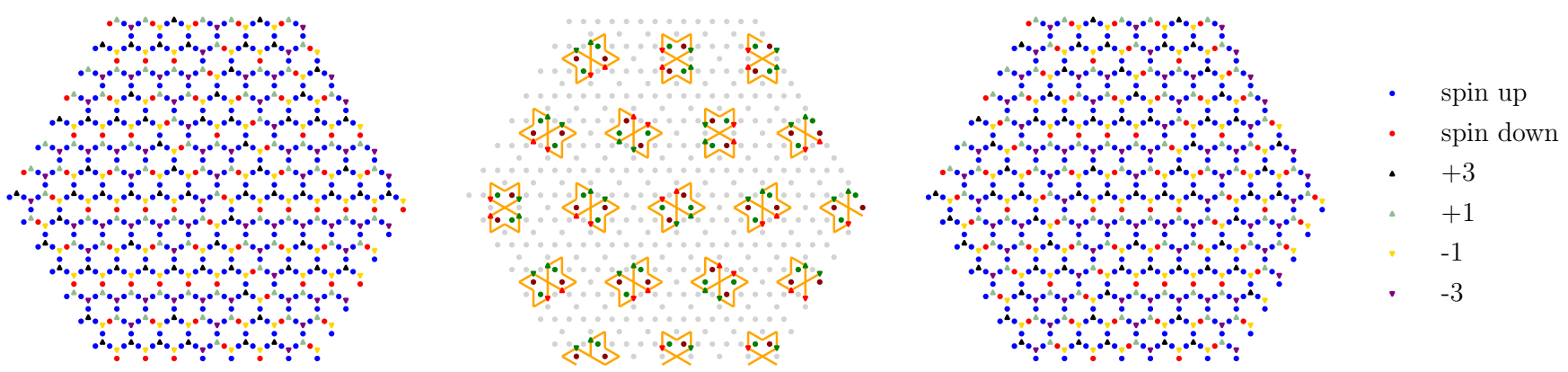

FIG. 33. Two examples of ground-state configurations for the $J_{1}-J_{2}-J_{3||}$ model, including the corresponding charge configuration, for a magnetic field $4\left(J_{1}+J_{2}\right)-2 J_{3 \|}<h<4\left(J_{1}+J_{2}+J_{3 \|}\right)(m=17 / 27)$. Local updates shown in the central panel bring the system from one configuration to the other (see the caption of Fig. 29 for details). 
Nevertheless, it shows that on the kagome lattice, the residual entropy gets lifted only very progressively-in contrast to the triangular lattice Ising antiferromagnet, for instance. In particular, we want to underline that unlike Refs. [31,87], the case where $J_{3 \|}<J_{2}$ is not at a phase boundary between two ground-state phases of the $J_{1}-J_{2}-J_{3||}$ model. It is all the more surprising that a finite residual entropy is preserved at some values of the magnetic field.
[1] R. F. Wang, C. Nisoli, R. S. Freitas, J. Li, W. McConville, B. J. Cooley, M. S. Lund, N. Samarth, C. Leighton, V. H. Crespi, and P. Schiffer, Artificial spin ice in a geometrically frustrated lattice of nanoscale ferromagnetic islands, Nature (London) 439, 303 (2006).

[2] C. Nisoli, R. Moessner, and P. Schiffer, Colloquium: Artificial spin ice: Designing and imaging magnetic frustration, Rev. Mod. Phys. 85, 1473 (2013).

[3] L. J. Heyderman and R. L. Stamps, Artificial ferroic systems: Novel functionality from structure, interactions and dynamics, J. Phys.: Condens. Matter 25, 363201 (2013).

[4] N. Rougemaille and B. Canals, Cooperative magnetic phenomena in artificial spin systems: Spin liquids, coulomb phase and fragmentation of magnetism - a colloquium, Eur. Phys. J. B 92, 62 (2019).

[5] S. H. Skjaervø, C. H. Marrows, R. L. Stamps, and L. J. Heyderman, Advances in artificial spin ice, Nat. Rev. Phys. 2, 14 (2020).

[6] H. Arava, P. M. Derlet, J. Vijayakumar, J. Cui, N. S. Bingham, A. Kleibert, and L. J. Heyderman, Computational logic with square rings of nanomagnets, Nanotechnology 29, 265205 (2018).

[7] F. Caravelli and C. Nisoli, Logical gates embedding in artificial spin ice, New J. Phys. 22, 103052 (2020).

[8] Z. Gu, M. E. Nowakowski, D. B. Carlton, R. Storz, M.-Y. Im, J. Hong, W. Chao, B. Lambson, P. Bennett, M. T. Alam, M. A. Marcus, A. Doran, A. Young, A. Scholl, P. Fischer, and J. Bokor, Sub-nanosecond signal propagation in anisotropyengineered nanomagnetic logic chains, Nat. Commun. 6, 6466 (2015).

[9] J. H. Jensen, E. Folven, and G. Tufte, Computation in artificial spin ice, Artificial Life Conf. Proc. 15, 3 (2018).

[10] P. Zhou, N. R. McDonald, A. J. Edwards, L. Loomis, C. D. Thiem, and J. S. Friedman, Reservoir computing with planar nanomagnet arrays, arXiv:2003.10948.

[11] J. Cui, T.-Y. Huang, Z. Luo, P. Testa, H. Gu, X.-Z. Chen, B. J. Nelson, and L. J. Heyderman, Nanomagnetic encoding of shape-morphing micromachines, Nature (London) 575, 164 (2019).

[12] X. Ke, J. Li, C. Nisoli, P. E. Lammert, W. McConville, R. F. Wang, V. H. Crespi, and P. Schiffer, Energy Minimization and ac Demagnetization in a Nanomagnet Array, Phys. Rev. Lett. 101, 037205 (2008).

[13] C. Nisoli, J. Li, X. Ke, D. Garand, P. Schiffer, and V. H. Crespi, Effective Temperature in an Interacting Vertex System: Theory and Experiment on Artificial Spin Ice, Phys. Rev. Lett. 105, 047205 (2010).

[14] A. Farhan, P. M. Derlet, A. Kleibert, A. Balan, R. V. Chopdekar, M. Wyss, J. Perron, A. Scholl, F. Nolting, and L. J. Heyderman, Direct Observation of Thermal Relaxation in Artificial Spin Ice, Phys. Rev. Lett. 111, 057204 (2013).

[15] A. Farhan, P. M. Derlet, A. Kleibert, A. Balan, R. V. Chopdekar, M. Wyss, L. Anghinolfi, F. Nolting, and L. J. Heyderman, Ex- ploring hyper-cubic energy landscapes in thermally active finite artificial spin-ice systems, Nat. Phys. 9, 375 (2013).

[16] J. P. Morgan, J. Akerman, A. Stein, C. Phatak, R. M. L. Evans, S. Langridge, and C. H. Marrows, Real and effective thermal equilibrium in artificial square spin ices, Phys. Rev. B 87, 024405 (2013).

[17] S. Zhang, I. Gilbert, C. Nisoli, G. W. Chern, M. J. Erickson, L. O'Brien, C. Leighton, P. E. Lammert, V. H. Crespi, and P. Schiffer, Crystallites of magnetic charges in artificial spin ice, Nature (London) 500, 553 (2013).

[18] U. B. Arnalds, M. Ahlberg, M. S. Brewer, V. Kapaklis, E. T. Papaioannou, M. Karimipour, P. Korelis, A. Stein, S. Ólafsson, T. P. A. Hase, and B. Hjörvarsson, Thermal transitions in nanopatterned XY-magnets, Appl. Phys. Lett. 105, 042409 (2014).

[19] V. Kapaklis, U. B. Arnalds, A. Farhan, R. V. Chopdekar, A. Balan, A. Scholl, L. J. Heyderman, and B. Hjörvarsson, Thermal fluctuations in artificial spin ice, Nat. Nanotechnol. 9, 514 (2014).

[20] G. Möller and R. Moessner, Magnetic multipole analysis of kagome and artificial spin-ice dipolar arrays, Phys. Rev. B 80, 140409(R) (2009).

[21] G.-W. Chern, P. Mellado, and O. Tchernyshyov, Two-Stage Ordering of Spins in Dipolar Spin Ice on the Kagome Lattice, Phys. Rev. Lett. 106, 207202 (2011).

[22] I. A. Chioar, N. Rougemaille, A. Grimm, O. Fruchart, E. Wagner, M. Hehn, D. Lacour, F. Montaigne, and B. Canals, Nonuniversality of artificial frustrated spin systems, Phys. Rev. B 90, 064411 (2014).

[23] I. A. Chioar, N. Rougemaille, and B. Canals, Ground-state candidate for the classical dipolar kagome Ising antiferromagnet, Phys. Rev. B 93, 214410 (2016).

[24] J. Hamp, R. Moessner, and C. Castelnovo, Supercooling and fragile glassiness in a dipolar kagome Ising magnet, Phys. Rev. B 98, 144439 (2018).

[25] L. F. Cugliandolo, L. Foini, and M. Tarzia, Mean-field phase diagram and spin-glass phase of the dipolar kagome Ising antiferromagnet, Phys. Rev. B 101, 144413 (2020).

[26] G. Möller and R. Moessner, Artificial Square Ice and Related Dipolar Nanoarrays, Phys. Rev. Lett. 96, 237202 (2006).

[27] Y. Perrin, B. Canals, and N. Rougemaille, Extensive degeneracy, Coulomb phase and magnetic monopoles in artificial square ice, Nature (London) 540, 410 (2016).

[28] A. Farhan, M. Saccone, C. F. Petersen, S. Dhuey, R. V. Chopdekar, Y.-L. Huang, N. Kent, Z. Chen, M. J. Alava, T. Lippert, A. Scholl, and S. van Dijken, Emergent magnetic monopole dynamics in macroscopically degenerate artificial spin ice, Sci. Adv. 5, aav6380 (2019).

[29] Z. Luo, T. P. Dao, A. Hrabec, J. Vijayakumar, A. Kleibert, M. Baumgartner, E. Kirk, J. Cui, T. Savchenko, G. Krishnaswamy et al., Chirally coupled nanomagnets, Science 363, 1435 (2019).

[30] S. Kempinger, Y.-S. Huang, P. Lammert, M. Vogel, A. Hoffmann, V. H. Crespi, P. Schiffer, and N. Samarth, 
Field-tunable interactions and frustration in underlayermediated artificial spin ice, arXiv:2104.00126.

[31] T. Mizoguchi, L. D. C. Jaubert, and M. Udagawa, Clustering of Topological Charges in a Kagome Classical Spin Liquid, Phys. Rev. Lett. 119, 077207 (2017).

[32] T. Nishino and K. Okunishi, Corner transfer matrix renormalization group method, J. Phys. Soc. Jpn. 65, 891 (1996).

[33] R. Orús, A practical introduction to tensor networks: Matrix product states and projected entangled pair states, Ann. Phys. 349, 117 (2014).

[34] J. Haegeman and F. Verstraete, Diagonalizing transfer matrices and matrix product operators: A medley of exact and computational methods, Annu. Rev. Condens. Matter Phys. 8, 355 (2017)

[35] U. Schollwöck, The density-matrix renormalization group in the age of matrix product states, Ann. Phys. 326, 96 (2011), January 2011 Special Issue.

[36] M. T. Fishman, L. Vanderstraeten, V. Zauner-Stauber, J. Haegeman, and F. Verstraete, Faster methods for contracting infinite two-dimensional tensor networks, Phys. Rev. B 98, 235148 (2018).

[37] M. Levin and C. P. Nave, Tensor Renormalization Group Approach to Two-Dimensional Classical Lattice Models, Phys. Rev. Lett. 99, 120601 (2007).

[38] Z. Y. Xie, J. Chen, M. P. Qin, J. W. Zhu, L. P. Yang, and T. Xiang, Coarse-graining renormalization by higher-order singular value decomposition, Phys. Rev. B 86, 045139 (2012).

[39] G. Evenbly and G. Vidal, Tensor Network Renormalization, Phys. Rev. Lett. 115, 180405 (2015).

[40] W. Li, S.-S. Gong, Y. Zhao, S.-J. Ran, S. Gao, and G. Su, Phase transitions and thermodynamics of the two-dimensional Ising model on a distorted kagome lattice, Phys. Rev. B 82, 134434 (2010).

[41] L. Vanderstraeten, B. Vanhecke, and F. Verstraete, Residual entropies for three-dimensional frustrated spin systems with tensor networks, Phys. Rev. E 98, 042145 (2018).

[42] B. Vanhecke, J. Colbois, L. Vanderstraeten, F. Verstraete, and F. Mila, Solving frustrated Ising models using tensor networks, Phys. Rev. Research 3, 013041 (2021).

[43] K. Kano and S. Naya, Antiferromagnetism. The kagome Ising net, Prog. Theor. Phys. 10, 158 (1953).

[44] Sütö, Models of superfrustration, Z. Phys. B: Condens. Matter 44, 121 (1981).

[45] J. H. Barry and M. Khatun, Exact solutions for correlations in the kagomé Ising antiferromagnet, Int. J. Mod. Phys. B 11, 93 (1997).

[46] A. S. Wills, R. Ballou, and C. Lacroix, Model of localized highly frustrated ferromagnetism: The kagomé spin ice, Phys. Rev. B 66, 144407 (2002).

[47] W. Apel and H.-U. Everts, Correlations in the Ising antiferromagnet on the anisotropic kagome lattice, J. Stat. Mech. (2011) P09002.

[48] K. A. Muttalib, M. Khatun, and J. H. Barry, Perpendicular susceptibility and geometrical frustration in two-dimensional Ising antiferromagnets: Exact solutions, Phys. Rev. B 96, 184411 (2017).

[49] I. Dzyaloshinsky, A thermodynamic theory of 'weak' ferromagnetism of antiferromagnetics, J. Phys. Chem. Solids 4, 241 (1958).
[50] T. Moriya, Anisotropic superexchange interaction and weak ferromagnetism, Phys. Rev. 120, 91 (1960).

[51] A. Fert, Magnetic and transport properties of metallic multilayers, in Metallic Multilayers, Materials Science Forum (Trans Tech Publications Ltd, Baech, Switzerland, 1991), Vol. 59, pp. 439-480.

[52] A. Crépieux and C. Lacroix, Dzyaloshinsky-Moriya interactions induced by symmetry breaking at a surface, J. Magn. Magn. Mater. 182, 341 (1998).

[53] N. Rougemaille, F. Montaigne, B. Canals, A. Duluard, D. Lacour, M. Hehn, R. Belkhou, O. Fruchart, S. El Moussaoui, A. Bendounan, and F. Maccherozzi, Artificial Kagome Arrays of Nanomagnets: A Frozen Dipolar Spin Ice, Phys. Rev. Lett. 106, 057209 (2011).

[54] M. Saccone, K. Hofhuis, Y.-L. Huang, S. Dhuey, Z. Chen, A. Scholl, R. V. Chopdekar, S. van Dijken, and A. Farhan, Dipolar Cairo lattice: Geometrical frustration and short-range correlations, Phys. Rev. Mater. 3, 104402 (2019).

[55] A. Farhan, M. Saccone, C. F. Petersen, S. Dhuey, K. Hofhuis, R. Mansell, R. V. Chopdekar, A. Scholl, T. Lippert, and S. van Dijken, Geometrical Frustration and Planar Triangular Antiferromagnetism in Quasi-Three-Dimensional Artificial Spin Architecture, Phys. Rev. Lett. 125, 267203 (2020).

[56] A. Vansteenkiste, J. Leliaert, M. Dvornik, M. Helsen, F. GarciaSanchez, and B. Van Waeyenberge, The design and verification of MuMax ${ }^{3}$, AIP Adv. 4, 107133 (2014).

[57] J. Leliaert, M. Dvornik, J. Mulkers, J. De Clercq, M. V. Milošević, and B. Van Waeyenberge, Fast micromagnetic simulations on GPU-recent advances made with mumax ${ }^{3}$, J. Phys. D 51, 123002 (2018).

[58] $J_{1, \mathrm{~h}}=1.868 \times 10^{-20} \mathrm{~J}$ while $J_{1, \mathrm{~d}}=1.884 \times 10^{-20} \mathrm{~J}$. We use the average of these values for $J_{1}$, and the difference of these values to the average as an estimate of the error on $J_{1}$.

[59] A careful look at the MFM results showed some islands changing contrast during the tip scanning process, which suggests a tip-sample interaction.

[60] R. Moessner, S. L. Sondhi, and P. Chandra, Two-Dimensional Periodic Frustrated Ising Models in a Transverse Field, Phys. Rev. Lett. 84, 4457 (2000).

[61] R. Moessner and S. L. Sondhi, Ising models of quantum frustration, Phys. Rev. B 63, 224401 (2001).

[62] M. Udagawa, M. Ogata, and Z. Hiroi, Exact result of groundstate entropy for Ising pyrochlore magnets under a magnetic field along [111] axis, J. Phys. Soc. Jpn. 71, 2365 (2002).

[63] R. Moessner and S. L. Sondhi, Theory of the [111] magnetization plateau in spin ice, Phys. Rev. B 68, 064411 (2003).

[64] I. Syozi, Statistics of kagome lattice, Prog. Theor. Phys. 6, 306 (1951).

[65] Note that the magnetization is zero in the ground state of the model, hence the connected or disconnected correlations have the same value.

[66] R. J. Baxter, Exactly Solved Models in Statistical Mechanics (Dover Publications Inc., New York, 2007), p. 36.

[67] V. Zauner-Stauber, L. Vanderstraeten, M. T. Fishman, F. Verstraete, and J. Haegeman, Variational optimization algorithms for uniform matrix product states, Phys. Rev. B 97, 045145 (2018).

[68] G. Rakala and K. Damle, Cluster algorithms for frustrated two-dimensional Ising antiferromagnets via dual worm constructions, Phys. Rev. E 96, 023304 (2017). 
[69] M. M. Rams, P. Czarnik, and L. Cincio, Precise Extrapolation of the Correlation Function Asymptotics in Uniform Tensor Network States with Application to the Bose-Hubbard and XXZ Models, Phys. Rev. X 8, 041033 (2018).

[70] As discussed in Ref. [4], the notion of effective temperature mostly describes whether a given snapshot of a configuration of the nanomagnets is characteristic of some state at equilibrium or, conversely, whether an out-of-equilibrium description is required. In the same spirit, it is now quite standard in artificial spin ices to consider an energy-based effective temperature corresponding to the canonical distribution describing effectively the vertex population [13]. This is the approach that we followed here.

[71] G. H. Wannier, Antiferromagnetism. The triangular Ising net, Phys. Rev. 79, 357 (1950).

[72] G. H. Wannier, Antiferromagnetism. the triangular Ising net, Phys. Rev. B 7, 5017 (1973).

[73] Y. L. Loh, D. X. Yao, and E. W. Carlson, Thermodynamics of Ising spins on the triangular kagome lattice: Exact analytical method and Monte Carlo simulations, Phys. Rev. B 77, 134402 (2008).

[74] B. D. Metcalf and C. P. Yang, Degeneracy of antiferromagnetic Ising lattices at critical magnetic field and zero temperature, Phys. Rev. B 18, 2304 (1978).

[75] C.-O. Hwang, S.-Y. Kim, D. Kang, and J. M. Kim, Ising antiferromagnets in a nonzero uniform magnetic field, J. Stat. Mech. (2007) L05001.

[76] M. Semjan and M. Žukovič, Magnetocaloric properties of an Ising antiferromagnet on a kagome lattice, Acta Phys. Pol. A 137, 622 (2020).

[77] We also confirmed our results by using a slightly different tensor network formulation based on ground state local rules in the spirit of Ref. [42].

[78] J. Colbois and K. Hofhuis, Open access data for the present paper (2021).

[79] K. Soldatov, A. Peretyatko, P. Andriushchenko, K. Nefedev, and Y. Okabe, Comparison of diluted antiferromagnetic Ising models on frustrated lattices in a magnetic field, Phys. Lett. A 383, 1229 (2019).

[80] T. Takagi and M. Mekata, Magnetic ordering of Ising spins on kagome lattice with the 1 st and the 2nd neighbor interactions, J. Phys. Soc. Jpn. 62, 3943 (1993).

[81] G.-W. Chern and O. Tchernyshyov, Magnetic charge and ordering in kagome spin ice, Philos. Trans. R. Soc. A 370, 5718 (2012).
[82] M. Wolf and K. D. Schotte, Ising model with competing nextnearest-neighbour interactions on the kagome lattice, J. Phys. A: Math. Gen. 21, 2195 (1988).

[83] Notice the opposite sign convention and factor two.

[84] M. Kaburagi and J. Kanamori, A method of determining the ground state of the extended-range classical lattice gas model, Prog. Theor. Phys. 54, 30 (1975).

[85] Z. Zhu and H. G. Katzgraber, Do tensor renormalization group methods work for frustrated spin systems? arXiv:1903.07721.

[86] J.-G. Liu, L. Wang, and P. Zhang, Tropical Tensor Network for Ground States of Spin Glasses, Phys. Rev. Lett. 126, 090506 (2021).

[87] K. Tokushuku, T. Mizoguchi, and M. Udagawa, Field-selective classical spin liquid and magnetization plateaus on kagome lattice, J. Phys. Soc. Jpn. 89, 053708 (2020).

[88] W. Huang, D. A. Kitchaev, S. T. Dacek, Z. Rong, A. Urban, S. Cao, C. Luo, and G. Ceder, Finding and proving the exact ground state of a generalized Ising model by convex optimization and MAX-SAT, Phys. Rev. B 94, 134424 (2016).

[89] Meaning the corresponding ground state tiles fail to tile the lattice and the tensor network contraction fails. Larger clusters would be required, probably made of 6 to 12 stars from what we see of the Monte Carlo simulations; this requires some adaptation of the code, which we did not yet tackle.

[90] This would perhaps give results similar to Eqs. (10).

[91] For instance, by combining X-PEEM with MFM.

[92] R. F. Wang, J. Li, W. McConville, C. Nisoli, X. Ke, J. W. Freeland, V. Rose, M. Grimsditch, P. Lammert, V. H. Crespi, and P. Schiffer, Demagnetization protocols for frustrated interacting nanomagnet arrays, J. Appl. Phys. 101, 09J104 (2007).

[93] V. M. Parakkat, K. Xie, and K. M. Krishnan, Tunable ground state in heterostructured artificial spin ice with exchange bias, Phys. Rev. B 99, 054429 (2019).

[94] K. Hukushima and K. Nemoto, Exchange Monte Carlo method and application to spin glass simulations, J. Phys. Soc. Jpn. 65, 1604 (1996).

[95] V. Ambegaokar and M. Troyer, Estimating errors reliably in Monte Carlo simulations of the Ehrenfest model, Am. J. Phys. 78, 150 (2010).

[96] F. Romá, F. Nieto, E. E. Vogel, and A. J. Ramirez-Pastor, Ground-state entropy of $\pm j$ Ising lattices by Monte Carlo simulations, J. Stat. Phys. 114, 1325 (2004). 